\title{
Thermomagnetic properties and Bjorken expansion of hot QCD matter in a strong magnetic field
}

\author{
Shubhalaxmi Rath* and Binoy Krishna Patra ${ }^{\dagger}$ \\ Department of Physics, \\ Indian Institute of Technology Roorkee, Roorkee 247667, India
}

\begin{abstract}
In this work we have studied the effects of an external strong magnetic field on the thermodynamic and magnetic properties of a hot QCD matter and then explored these effects on the subsequent hydrodynamic expansion of the said matter once produced in the ultrarelativistic heavy ion collisions. For that purpose, we have computed the quark and gluon self-energies up to one loop in the strong magnetic field, using the HTL approximation with two hard scales - temperature and magnetic field, which in turn compute the effective propagators for quarks and gluons, respectively. Hence the quark and gluon contributions to the free energy are obtained from the respective propagators and finally derive the equation of state (EOS) by calculating the pressure and energy density. We have found that the speed of sound is enhanced due to the presence of strong magnetic field and this effect will be later exploited in the hydrodynamics. Thereafter the magnetic properties are studied from the free energy of the matter, where the magnetization is found to increase linearly with the magnetic field, thus hints the paramagnetic behavior. The temperature dependence of the magnetization is also studied within the strong magnetic field limit $\left(\left|q_{f} B\right| \gg T^{2},\left|q_{f} B\right| \gg m_{f}^{2}\right.$, where $\left|q_{f}\right|\left(m_{f}\right)$ is the absolute electric charge (mass) of $f$-th flavor), where the magnetization is found to increase slowly with the temperature. Finally, to see how a strong magnetic field could affect the hydrodynamic evolution, we have revisited the Bjorken boost-invariant picture with our paramagnetic EOS as an input in the equation of motion for the energy-momentum conservation. We have noticed that the energy density evolves faster than in the absence of strong magnetic field, i.e. cooling becomes faster, which could have implications on the heavy-ion phenomenology. As mentioned earlier, this observation can be understood by the enhancement of the speed of sound.
\end{abstract}

Keywords: Self energy; Free energy; Equation of state; Strong magnetic field; Magnetization; Susceptibility; Bjorken expansion.

*srath@ph.iitr.ac.in

†binoyfph@iitr.ac.in 


\section{Introduction}

The possibility to achieve strong magnetic fields at the noncentral events of ultrarelativistic heavy ion collisions (URHIC) [1,2] viz. at RHIC, LHC, makes it feasible in reality to explore the thermodynamic and magnetic properties of QCD matter in the presence of both heat-bath and strong magnetic field. Recently the lattice QCD simulations have determined the equation of state (EOS) for a $(2+1)$-flavor QCD in the presence of magnetic fields by calculating the thermodynamic observables $[3,4]$. Similarly the effects of strong magnetic fields on the phase structures of the hadronic matter in the framework of effective theories have also been reviewed in [5]. We have very recently investigated the thermodynamic observables for the same perturbatively in a strong magnetic field (SMF) limit [6]. Like the thermodynamic properties, the magnetic properties of a hot QCD matter might be modified due to the presence of strong magnetic field. Different materials placed in an external magnetic field evince different responses to the magnetic field and depending on the responses, known as magnetization, they are classified as paramagnets and diamagnets. Paramagnetic response is defined by the positive value of the magnetization, i.e. the case when the free energy of the system decreases due to the magnetic field, whereas the negative value of the magnetization defines the system or material as a diamagnet. The classification of magnetic materials can be alternatively described in terms of magnetic susceptibility. So the magnetic properties of QCD matter too can also be envisaged by its response to the external magnetic field and can thus be understood by the free energy of the system, which in general embraces all the informations about the magnetic and thermodynamic properties of a system. The knowledge about the magnetic response of the QCD matter helps greatly in exploring the chiral magnetic effect $[7,8]$, the photon production rate from the hot QCD medium $[9,10]$, the dilepton production from the QGP [11, 12], the collective expansion of the fireball [13], the axial magnetic field $[14,15]$, the chiral vortical effect [16], the (inverse) magnetic catalysis arising due to the (restoration) breaking of the chiral symmetry [17-19], the bulk properties of a Fermi gas [20], the production of soft photons in heavy ion collisions [21, 22], the Faraday rotation in a magnetized thermal medium $[23,24]$ and the electromagnetic radiation [25]. The magnetic response of QCD matter behaves as a theoretical tool in the area of high energy physics where strong magnetic fields might exist, such as the beginning of the universe and the cores of the magnetars, in addition to RHIC and LHC.

Thus we get motivated to analyse the magnetic response of a hot QCD matter in an ambience of strong magnetic field, which have been studied previously in different effective 
theories, such as in the potential model [26], the holographic QCD [27], Fermi-liquid theory by evaluating the spin susceptibility [28], the transport model [29], the linear sigma model with the Polyakov loop [30] and the quark-meson model applying the functional renormalization group method [31]. The above lattice study [3] also investigates the effect of background magnetic field on the magnetization from the free energy. However, our aim is to find the effects of strong magnetic field on the magnetic properties of the hot QCD matter as well as on the dynamical evolution of the same matter. So we have first revisited the free energy of the aforesaid matter in strong magnetic field $\left(\left|q_{f} B\right| \gg T^{2},\left|q_{f} B\right| \gg m_{f}^{2}\right)$ perturbatively up to one loop in HTL approximation with two hard scales - one is the temperature for gluons and the other is the strong magnetic field for quarks. The free energy further derives the EOS, where we have found that the square of the speed of sound gets enhanced due to the presence of strong magnetic field. This observation will be exploited in the expansion of the matter. We will next study the magnetic properties through the magnetization and magnetic susceptibility, which are obtained from the abovementioned perturbative free energy. We have found that the magnetization thus obtained indicates the paramagnetic nature of the hot QCD matter.

So far we have discussed the thermodynamic and magnetic properties of the medium, assuming the medium as static. But the medium produced in the ultrarelativistic heavy ion collisions immediately expands hydrodynamically. The hydrodynamic expansion in the presence of magnetic field has been studied in a longitudinal boost-invariant Bjorken picture by comparing the energy densities associated with the magnetic field and the fluid in event-by-event basis $[32,33]$. Using the $(3+1)$-dimensional anomalous relativistic hydrodynamics [34], it has been shown that the chiral magnetic effect is present in the charge dependent hadron azimuthal correlations. Similarly using the $(3+1)$-dimensional ideal hydrodynamics, the effect of spatially inhomogeneous magnetic field on the anisotropic expansion of the QGP in heavy ion collision has been studied in [35]. There are also recent studies of the hydrodynamic expansion in the presence of magnetic field by parameterizing the magnetic nature of the matter, i.e. the EOS with nonzero magnetization $[36,37]$. The abovementioned studies dealt with the conservation of energy-momentum tensor by incorporating the effect of magnetic field in it, known as relativistic magnetohydrodynamics. As our second aim, we will examine the same effects on the expansion through the EOS of the hot QCD matter, which is of paramagnetic nature, as an input in the hydrodynamical equation of motion, unlike the previous studies. Contrary to the above $[36,37]$, we have derived the EOS from the free energy and checked its magnetic character, then plugged in to the hydrodynamics directly. As found earlier, the square of 
speed of sound $\left(c_{s}^{2}\right)$ gets enhanced due to the effects of strong magnetic field, the energy density is consequently found to evolve faster than in the absence of strong magnetic field, implying that the matter now cools off faster.

The paper is organized in the following way. In section 2 , we have evaluated the free energies due to quark and gluon contributions from the respective effective propagators and obtained the equation of state. Using the free energies thus obtained, we have studied the magnetic properties of the QCD medium in the strong magnetic field by determining the magnetization and magnetic susceptibility in section 3. Then in section 4, we proceeded to observe the Bjorken expansion in the strong magnetic field regime. We concluded in section 5. Finally, in appendices A, B and $\mathrm{C}$ we have provided complete derivations of the quark self-energy, the quark contribution to the free energy and integrals in the presence of a strong magnetic field, respectively.

\section{Free energy}

The one-loop free energy $(\mathcal{F})$ of a system of interacting quarks and gluons is the sum of free energies due to quarks and gluons, i.e. $\mathcal{F}=\mathcal{F}_{q}+\mathcal{F}_{g}$. In the coming subsections, we are going to discuss these contributions broadly.

\subsection{Quark contribution to the free energy}

The quark contribution $\left(\mathcal{F}_{q}\right)$ is calculated perturbatively in the fundamental representation of $\mathrm{SU}\left(N_{c}\right)$ gauge theory by the functional determinant of the effective quark propagator, $S(P)$,

$$
\mathcal{F}_{q}=N_{c} N_{f} \int \frac{d^{4} P}{(2 \pi)^{4}} \ln [\operatorname{det}(S(P))],
$$

where $S(P)$ is obtained by the one-loop quark self-energy in a strong magnetic field through the Schwinger-Dyson equation,

$$
S^{-1}(P)=S_{0}^{-1}(P)-\Sigma(P) .
$$

The quark propagator was first obtained by Schwinger through the proper-time method [38] in the coordinate-space,

$$
S\left(x, x^{\prime}\right)=\phi\left(x, x^{\prime}\right) \int \frac{d^{4} K}{(2 \pi)^{4}} e^{-i K\left(x-x^{\prime}\right)} S(K),
$$


where the gauge-dependent phase factor, $\phi\left(x, x^{\prime}\right)$ is written in terms of path-representation of gauge fields,

$$
\phi\left(x, x^{\prime}\right)=e^{i\left|q_{f}\right| \int_{x^{\prime}}^{x} A^{\mu}(\zeta) d \zeta_{\mu}} .
$$

However, by making an appropriate gauge transformation, the phase factor can be gauged away. In fact, for a single fermion line, the phase factor in a symmetric gauge becomes unity, hence the propagator can also be written in the momentum-space as an integral over the Schwinger proper-time $(s)$,

$$
\begin{aligned}
S(K)= & i \int_{0}^{\infty} d s \exp \left(i s k_{\|}^{2}-i s m_{f}^{2}-\frac{i k_{\perp}^{2} \tan \left(\left|q_{f} B\right| s\right)}{\left|q_{f} B\right|}\right) \\
& \times\left\{\left(m_{f}+\gamma^{\|} \cdot k_{\|}\right)\left(1+\gamma^{1} \gamma^{2} \tan \left(\left|q_{f} B\right| s\right)\right)-\gamma^{\perp} \cdot k_{\perp}\left(1+\tan ^{2}\left(\left|q_{f} B\right| s\right)\right)\right\},
\end{aligned}
$$

where the notations are given by

$$
\begin{aligned}
& k_{\|} \equiv\left(k_{0}, 0,0, k_{3}\right), \quad k_{\perp} \equiv\left(0, k_{1}, k_{2}, 0\right), \quad \gamma^{\|} \equiv\left(\gamma^{0}, \gamma^{3}\right), \quad \gamma^{\perp} \equiv\left(\gamma^{1}, \gamma^{2}\right), \\
& g_{\|}^{\mu \nu}=\operatorname{diag}(1,0,0,-1), \quad g_{\perp}^{\mu \nu}=\operatorname{diag}(0,-1,-1,0), \quad g^{\mu \nu}=g_{\|}^{\mu \nu}+g_{\perp}^{\mu \nu} .
\end{aligned}
$$

In terms of discretized Landau levels, $S(K)$ can be written in a discrete notation,

$$
S(K)=i e^{-\frac{k_{\perp}^{2}}{\left|q_{f} B\right|}} \sum_{n=0}^{\infty}(-1)^{n} \frac{D_{n}\left(\left|q_{f} B\right|, K\right)}{k_{\|}^{2}-m_{f}^{2}-2\left|q_{f} B\right| n},
$$

where $D_{n}$ 's are expressed in terms of the Laguerre polynomials, $L_{n}$ 's [39] as

$$
\begin{aligned}
D_{n}\left(\left|q_{f} B\right|, K\right)= & \left(\gamma^{\|} \cdot k_{\|}+m_{f}\right)\left[\left(1-i \gamma^{1} \gamma^{2}\right) L_{n}\left(\frac{2 k_{\perp}^{2}}{\left|q_{f} B\right|}\right)-\left(1+i \gamma^{1} \gamma^{2}\right) L_{n-1}\left(\frac{2 k_{\perp}^{2}}{\left|q_{f} B\right|}\right)\right] \\
& +4 \gamma^{\perp} \cdot k_{\perp} L_{n-1}^{(1)}\left(\frac{2 k_{\perp}^{2}}{\left|q_{f} B\right|}\right) .
\end{aligned}
$$

In a strong magnetic field, only the lowest Landau level (LLL) $(n=0)$ is occupied, hence the quark propagator (6) simplifies into

$$
S_{0}(K)=i e^{-\frac{k_{\perp}^{2}}{\left|q_{f} B\right|}} \frac{\left(\gamma^{\|} \cdot k_{\|}+m_{f}\right)}{k_{\|}^{2}-m_{f}^{2}+i \epsilon}\left(1-\gamma^{0} \gamma^{3} \gamma^{5}\right) .
$$

Since the gluons are electrically uncharged particles, they are not directly affected by the Lorentz force, therefore the form of vacuum propagator for gluon remains the same, i.e.

$$
D_{0}^{\mu \nu}(Q)=\frac{i g^{\mu \nu}}{Q^{2}+i \epsilon}
$$


In a thermal medium, both the Schwinger's proper-time propagator for quark (8) and the gluon propagator $(9)$ get thermalized in the real-time formalism by the following $2 \times 2$ matrices,

$$
\begin{aligned}
S(K) & =\left(\begin{array}{cc}
n_{F}^{\prime \prime 2} S_{0}(K)-n_{F}^{\prime 2} S_{0}^{*}(K) & -n_{F}^{\prime} n_{F}^{\prime \prime}\left(S_{0}(K)+S_{0}^{*}(K)\right) \\
n_{F}^{\prime} n_{F}^{\prime \prime}\left(S_{0}(K)+S_{0}^{*}(K)\right) & n_{F}^{\prime \prime 2} S_{0}^{*}(K)-n_{F}^{\prime 2} S_{0}(K)
\end{array}\right), \\
D^{\mu \nu}(Q) & =\left(\begin{array}{cc}
n_{B}^{\prime 2} D_{0}^{* \mu \nu}(Q)+n_{B}^{\prime \prime 2} D_{0}^{\mu \nu}(Q) & n_{B}^{\prime} n_{B}^{\prime \prime}\left(D_{0}^{\mu \nu}(Q)+D_{0}^{* \mu \nu}(Q)\right) \\
n_{B}^{\prime} n_{B}^{\prime \prime}\left(D_{0}^{\mu \nu}(Q)+D_{0}^{* \mu \nu}(Q)\right) & n_{B}^{\prime 2} D_{0}^{\mu \nu}(Q)+n_{B}^{\prime \prime 2} D_{0}^{* \mu \nu}(Q)
\end{array}\right),
\end{aligned}
$$

respectively. The above notations are redefined in terms of quark $\left(n_{F}\right)$ and gluon $\left(n_{B}\right)$ equilibrium distribution functions as $n_{F}^{\prime}=\sqrt{n_{F}\left(k_{0}\right)}, n_{F}^{\prime \prime}=\sqrt{1-n_{F}\left(k_{0}\right)}, n_{B}^{\prime}=\sqrt{n_{B}\left(q_{0}\right)}$ and $n_{B}^{\prime \prime}=\sqrt{1+n_{B}\left(q_{0}\right)}$.

For the description of a system in equilibrium quantum field theory, only the 11component of the self-energy matrix is sufficient, so we need only the 11-components of the quark and gluon propagators in a strongly magnetized thermal medium to calculate the respective effective propagators, which are therefore given by (from matrices (10) and $(11))$

$$
\begin{aligned}
S_{11}(K)= & i e^{-\frac{k_{\perp}^{2}}{\left|q_{f} B\right|}\left(\gamma^{0} k_{0}-\gamma^{3} k_{3}+m_{f}\right)\left(1-\gamma^{0} \gamma^{3} \gamma^{5}\right)} \\
& \times\left[\frac{1}{k_{\|}^{2}-m_{f}^{2}+i \epsilon}+2 \pi i n_{F}\left(k_{0}\right) \delta\left(k_{\|}^{2}-m_{f}^{2}\right)\right], \\
D_{11}^{\mu \nu}(Q)= & i g^{\mu \nu}\left[\frac{1}{Q^{2}+i \epsilon}-2 \pi i n_{B}\left(q_{0}\right) \delta\left(Q^{2}\right)\right],
\end{aligned}
$$

respectively. The temperature dependences are contained in the quark and gluon distribution functions,

$$
\begin{aligned}
& n_{F}\left(k_{0}\right)=\frac{1}{e^{\beta\left|k_{0}\right|}+1}, \\
& n_{B}\left(q_{0}\right)=\frac{1}{e^{\beta\left|q_{0}\right|}-1},
\end{aligned}
$$

respectively with $T=1 / \beta$.

Thus the 11-component of one-loop quark self-energy (omitting the subscript "11") in terms of the 11-components of the quark (12) and gluon (13) propagators in a strong 
magnetic field is written as

$$
\begin{aligned}
\Sigma(P)= & -\frac{4}{3} g^{2} i \int \frac{d^{4} K}{(2 \pi)^{4}}\left[\gamma_{\mu} S_{11}(K) \gamma^{\mu} D_{11}(P-K)\right] \\
= & \frac{4}{3} \frac{g^{2} i}{(2 \pi)^{4}} \int d^{2} k_{\perp} d^{2} k_{\|} e^{-\frac{k_{\perp}^{2}}{\left|q_{f} B\right|}}\left[\gamma_{\mu}\left(\gamma^{0} k_{0}-\gamma^{3} k_{3}+m_{f}\right)\left(1-\gamma^{0} \gamma^{3} \gamma^{5}\right) \gamma^{\mu}\right] \\
& \times\left[\frac{1}{k_{\|}^{2}-m_{f}^{2}+i \epsilon}+2 \pi i n_{F}\left(k_{0}\right) \delta\left(k_{\|}^{2}-m_{f}^{2}\right)\right] \\
& \times\left[\frac{1}{(P-K)^{2}+i \epsilon}-2 \pi i n_{B}\left(p_{0}-k_{0}\right) \delta\left((P-K)^{2}\right)\right],
\end{aligned}
$$

where the factor, 4/3 represents the QCD colour factor and $g\left(g^{2}=4 \pi \alpha_{s}(e B)\right)$ is the running QCD coupling, that runs with the magnetic field because the magnetic field is the dominant energy scale of the thermal medium in a strong magnetic field $\left(e B \gg T^{2}\right)$, similar to the thermal medium in the absence of magnetic field where temperature is the dominant scale [40, 41]. The trace of gamma matrices in eq. (16) is calculated as

$$
\gamma_{\mu}\left(\gamma^{0} k_{0}-\gamma^{3} k_{3}+m_{f}\right)\left(1-\gamma^{0} \gamma^{3} \gamma^{5}\right) \gamma^{\mu}=-2\left[J_{+}\left(\gamma^{0} k_{0}-\gamma^{3} k_{3}\right)-2 m_{f}\right],
$$

with $J_{+}=1+\gamma^{0} \gamma^{3} \gamma^{5}$.

The quark self-energy (16) contains both the vacuum and medium contributions with single and product of two distribution functions. In the strong magnetic field (SMF) limit, due to the LLL approximation, the external quark momentum $P\left(p_{\|}, p_{\perp}\right)$ is assumed to be purely longitudinal [42], i.e. $p_{\perp}=0$. In addition, for the loop-momentum, both $k_{\|}^{2}$ and $k_{\perp}^{2}$ are much smaller than $\left|q_{f} B\right|$, so the form-factor, $e^{-k_{\perp}^{2} /\left|q_{f} B\right|}$ is approximated to be one. Thus the aforesaid artefacts of SMF limit facilitate to compute the real-part of the quark self-energy (in appendix A) as,

$$
\begin{aligned}
\Sigma\left(p_{\|}\right)= & \frac{J_{+}\left(\gamma^{\|} \cdot p_{\|}\right) g^{2}}{12 \pi^{2}}\left[-1-\frac{\left|q_{f} B\right|}{m_{f}^{2}}\left\{\ln \left(\frac{\left|q_{f} B\right|}{m_{f}^{2}}\right)-1\right\}\right] \\
& +\frac{g^{2} m_{f}}{3 \pi^{2}}\left[1+\frac{J_{+}\left|q_{f} B\right|}{2 m_{f}^{2}}\left\{\ln \left(\frac{\left|q_{f} B\right|}{m_{f}^{2}}\right)-1\right\}\right] \\
& -\frac{2 g^{2} m_{f}}{3 \pi^{2}} \ln \left(\frac{\left|q_{f} B\right|}{p_{\|}^{2}+m_{f}^{2}}\right)\left[\ln \left(\frac{m_{f}}{\pi T}\right)+\gamma_{E}\right],
\end{aligned}
$$

which in turn evaluates the effective quark propagator from the Schwinger-Dyson equation (2). 
Therefore the quark contribution to the free energy in a strong magnetic field is obtained from eq. (1) (in appendix B),

$$
\begin{aligned}
\mathcal{F}_{q} & =-\frac{N_{c} N_{f}\left|q_{f} B\right|}{4}\left[\frac{T^{2}}{3}+\int_{0}^{\left|q_{f} B\right|} \frac{d p_{\|}^{2}}{4 \pi^{2}} \ln \left[\left\{(1-2 \eta)^{2}-\frac{1}{p_{\|}^{2}}\left[m_{f}+2 \Gamma+\xi\right.\right.\right.\right. \\
& \left.\left.\left.+\lambda \ln \left(\frac{\left|q_{f} B\right|}{p_{\|}^{2}+m_{f}^{2}}\right)\right]^{2}\right\}\left\{1-\frac{1}{p_{\|}^{2}}\left[m_{f}+\xi+\lambda \ln \left(\frac{\left|q_{f} B\right|}{p_{\|}^{2}+m_{f}^{2}}\right)\right]^{2}\right\}\right],
\end{aligned}
$$

where the new variables are defined as

$$
\begin{aligned}
& \eta=-\frac{g^{2}}{12 \pi^{2}}\left[1+\frac{\left|q_{f} B\right|}{m_{f}^{2}}\left\{\ln \left(\frac{\left|q_{f} B\right|}{m_{f}^{2}}\right)-1\right\}\right], \quad \Gamma=\frac{g^{2}}{6 \pi^{2}} \frac{\left|q_{f} B\right|}{m_{f}}\left\{\ln \left(\frac{\left|q_{f} B\right|}{m_{f}^{2}}\right)-1\right\}, \\
& \xi=\frac{g^{2} m_{f}}{3 \pi^{2}}, \quad \lambda=-\frac{2 g^{2} m_{f}}{3 \pi^{2}}\left[\ln \left(\frac{m_{f}}{\pi T}\right)+\gamma_{E}\right] .
\end{aligned}
$$

\subsection{Gluon contribution to the free energy}

To calculate the free energy due to the gluon contribution $\left(\mathcal{F}_{g}\right)$ at finite temperature and finite magnetic field, we need to know the form of gluon self-energy tensor in the similar

environment. We will revisit here how to construct the general form of the gluon selfenergy tensor $\left(\Pi^{\mu \nu}\right)$ for an QCD medium at finite $T$ and finite $B$. Let us start with the vacuum, where the covariant form for the gluon self-energy tensor is given by

$$
\Pi^{\mu \nu}(p)=\left(g^{\mu \nu}-\frac{p^{\mu} p^{\nu}}{p^{2}}\right) \Pi\left(p^{2}\right),
$$

where $\Pi^{\mu \nu}$ satisfies the transversality condition. Now bring the vacuum into the direct contact of a heat reservoir $\left(u^{\mu}\right)$, so the Lorentz invariance is broken. Hence, a larger tensor basis is needed and the two orthogonal tensorial basis for finite temperature, $P_{T}^{\mu \nu}$ and $P_{L}^{\mu \nu}$ decompose the self-energy tensor [43-45] into

$$
\Pi^{\mu \nu}\left(p_{0}, \mathbf{p}\right)=\Pi_{T} P_{T}^{\mu \nu}\left(p_{0}, \mathbf{p}\right)+\Pi_{L} P_{L}^{\mu \nu}\left(p_{0}, \mathbf{p}\right)
$$

where

$$
\begin{aligned}
& P_{T}^{\mu \nu}=-g^{\mu \nu}+\frac{p_{0}}{\mathbf{p}^{2}}\left(p^{\mu} u^{\nu}+u^{\mu} p^{\nu}\right)-\frac{1}{\mathbf{p}^{2}}\left(p^{\mu} p^{\nu}+p^{2} u^{\mu} u^{\nu}\right), \\
& P_{L}^{\mu \nu}=-\frac{p_{0}}{\mathbf{p}^{2}}\left(p^{\mu} u^{\nu}+u^{\mu} p^{\nu}\right)+\frac{1}{\mathbf{p}^{2}}\left(\frac{p_{0}^{2}}{p^{2}} p^{\mu} p^{\nu}+p^{2} u^{\mu} u^{\nu}\right),
\end{aligned}
$$

are constructed in such a way that they satisfy the transversality condition $-p_{\mu} P_{T, L}^{\mu \nu}=0$. The subscripts $T$ and $L$ are said to be transverse and longitudinal with respect to the 
three-momentum $(\mathbf{p})$, respectively. So the corresponding structure factors, $\Pi_{T}$ and $\Pi_{L}$ are known as transverse and longitudinal components of self-energy, which are obtained in hard thermal loop (HTL) approximation, where the hard scale for both quark and gluon loop momenta $(p)$ is taken as the temperature $(p \sim T)$, justifying the name HTL. As a result, both quark and gluon loops give the same result $\sim \mathcal{O}\left(g^{2} T^{2}\right)$ apart from flavor and colour factors, respectively.

Now we introduce the magnetic field in a direction, $b^{\mu}$, to the thermal medium. So, the remaining translation invariance is broken and much larger basis is needed, which can be constructed with the vectors, $p^{\mu}, u^{\mu}, b^{\mu}$ and the tensor, $g^{\mu \nu}$. Therefore, in addition to the earlier ones, two new orthogonal tensors, $P_{\|}^{\mu \nu}$ and $P_{\perp}^{\mu \nu}$ are constructed for finite magnetic field [46, 47], at least, for the leading-order perturbation theory,

$$
\begin{gathered}
P_{\|}^{\mu \nu}=\frac{p_{0} p_{3}}{p_{\|}^{2}}\left(b^{\mu} u^{\nu}+u^{\mu} b^{\nu}\right)+\frac{1}{p_{\|}^{2}}\left(p_{0}^{2} b^{\mu} b^{\nu}+p_{3}^{2} u^{\mu} u^{\nu}\right), \\
P_{\perp}^{\mu \nu}=\frac{1}{p_{\perp}^{2}}\left[-p_{\perp}^{2} g^{\mu \nu}+p_{0}\left(p^{\mu} u^{\nu}+u^{\mu} p^{\nu}\right)+p_{3}\left(p^{\mu} b^{\nu}+b^{\mu} p^{\nu}\right)-p_{0} p_{3}\left(b^{\mu} u^{\nu}+u^{\mu} b^{\nu}\right)\right. \\
\left.\quad-p^{\mu} p^{\nu}+\left(p_{\perp}^{2}-p_{0}^{2}\right) u^{\mu} u^{\nu}-\left(p_{\perp}^{2}+p_{3}^{2}\right) b^{\mu} b^{\nu}\right],
\end{gathered}
$$

by demanding the same transversality condition. The notations used in above equations are defined as follows:

$$
\begin{aligned}
& u^{\mu}=(1,0,0,0), b^{\mu}=(0,0,0,-1), g_{\|}^{\mu \nu}=\operatorname{diag}(1,0,0-1), \\
& g_{\perp}^{\mu \nu}=\operatorname{diag}(0,-1,-1,0), p_{\|}^{2}=p_{0}^{2}-p_{3}^{2}, p_{\perp}^{2}=p_{1}^{2}+p_{2}^{2} .
\end{aligned}
$$

Therefore, the gluon self-energy tensor at finite $T$ and $B$ can be written as the superposition of finite $T((22),(23))$ and finite $B$ basis $((24),(25))$,

$$
\Pi^{\mu \nu}=\Pi_{T} P_{T}^{\mu \nu}+\Pi_{L} P_{L}^{\mu \nu}+\Pi_{\|} P_{\|}^{\mu \nu}+\Pi_{\perp} P_{\perp}^{\mu \nu},
$$

with the two new structure factors, $\Pi_{\|}$and $\Pi_{\perp}$ for finite $B$.

Now the entire batch of structure factors, $\Pi_{i}$ 's $(i=T, L, \|, \perp)$ can be evaluated in HTL approximation even in the presence of strong magnetic field $\left(e B \gg T^{2}\right.$ as well as $e B \gg m^{2}$ ), but with two hard scales, one is temperature and other is strong magnetic field perceived by gluons and quarks, respectively. More specifically, the temperature remains the hard scale for the gluon-loop momentum because gluons are not affected by the magnetic field but for the quark-loop momenta the hard scale is now replaced by the strong magnetic field $(\sqrt{e B})$ only due to SMF limit $\left(e B \gg T^{2}\right)$. Thus the gluon self-energy diagrams at finite $T$ and strong $B$ have been bifurcated into quark and gluon (ghost as well) loops, which give the thermal and magnetic field contributions, respectively. 


\subsubsection{Quark-loop contribution}

The quark-loop contribution will be obtained through $\Pi_{\|}$and $\Pi_{\perp}$. But for the strong magnetic field, the quarks are constrained to be in the lowest Landau levels and the dispersion relation ensures the perpendicular component (with respect to the magnetic field) to vanish, i.e. $p_{\perp} \approx 0$, which has been manifested by the vanishing structure factor, $\left(\Pi_{\perp} \approx 0\right)[46,48,49]$. Thus we are left to evaluate $\Pi_{\|}$.

In real-time formalism, using the LLL quark propagator (12), the 11-component of the gluon self-energy matrix (omitting the subscript "11") for the quark-loop is written as

$$
\begin{aligned}
\Pi_{q}^{\mu \nu}(P)= & -\frac{i g^{2}}{2} \int \frac{d^{4} K}{(2 \pi)^{4}} \operatorname{tr}\left[\gamma^{\mu} S_{11}(K) \gamma^{\nu} S_{11}(K-P)\right] \\
= & \frac{i g^{2}}{2} \sum_{f} \int \frac{d^{2} k_{\perp}}{(2 \pi)^{2}} \frac{d^{2} k_{\|}}{(2 \pi)^{2}} \operatorname{tr}\left[\gamma^{\mu}\left(\gamma^{0} k_{0}-\gamma^{3} k_{3}+m_{f}\right)\left(1-\gamma^{0} \gamma^{3} \gamma^{5}\right)\right. \\
& \left.\times \gamma^{\nu}\left(\gamma^{0} q_{0}-\gamma^{3} q_{3}+m_{f}\right)\left(1-\gamma^{0} \gamma^{3} \gamma^{5}\right)\right] e^{-\frac{k_{\perp}^{2}}{\left|q_{f} B\right|} e^{-\frac{q_{\perp}^{2}}{\left|q_{f} B\right|}}} \\
& \times\left[\frac{1}{k_{\|}^{2}-m_{f}^{2}+i \epsilon}+2 \pi i n_{F}\left(k_{0}\right) \delta\left(k_{\|}^{2}-m_{f}^{2}\right)\right] \\
& \times\left[\frac{1}{q_{\|}^{2}-m_{f}^{2}+i \epsilon}+2 \pi i n_{F}\left(q_{0}\right) \delta\left(q_{\|}^{2}-m_{f}^{2}\right)\right] \\
= & C_{\perp}\left(p_{\perp}\right) \Pi_{q}^{\mu \nu}\left(p_{\|}\right)
\end{aligned}
$$

where the factor $1 / 2$ accounts for the trace over colour indices. Since the magnetic field decouples the transverse dynamics from the longitudinal one, the gluon self-energy in LLL is manifestly separated into transverse-momentum dependent and longitudinal-momentum dependent parts. The transverse-momentum dependent part is thus easily calculated as

$$
C_{\perp}\left(p_{\perp}\right)=\sum_{f} \frac{\left|q_{f} B\right|}{8 \pi} e^{-\frac{p_{\perp}^{2}}{2\left|q_{f} B\right|}} .
$$

In the SMF limit, where $e^{-\frac{p_{\perp}^{2}}{2\left|q_{f} B\right|}} \approx 1$, the above equation is reduced into

$$
C_{\perp} \simeq \sum_{f} \frac{\left|q_{f} B\right|}{8 \pi}
$$

Next calculating the trace over gamma matrices,

$$
L^{\mu \nu}=8\left[k_{\|}^{\mu} \cdot q_{\|}^{\nu}+k_{\|}^{\nu} \cdot q_{\|}^{\mu}-g_{\|}^{\mu \nu}\left(k_{\|}^{\mu} \cdot q_{\| \mu}-m_{f}^{2}\right)\right]
$$


the longitudinal-momentum dependent part can be written as the superposition of three terms,

$$
\Pi_{q}^{\mu \nu}\left(p_{\|}\right)=\Pi_{q, V}^{\mu \nu}\left(p_{\|}\right)+\Pi_{q, n}^{\mu \nu}\left(p_{\|}\right)+\Pi_{q, n^{2}}^{\mu \nu}\left(p_{\|}\right)
$$

The vacuum contribution in eq. (31) is decomposed as

$$
\Pi_{q, V}^{\mu \nu}\left(p_{\|}\right)=\frac{i g^{2}}{8 \pi^{2}} \int d k_{0} d k_{3} \frac{L^{\mu \nu}}{\left(k_{\|}^{2}-m_{f}^{2}+i \epsilon\right)\left(q_{\|}^{2}-m_{f}^{2}+i \epsilon\right)},
$$

whose real part for the massless flavors yields to

$$
\Re \Pi_{q, V}^{\mu \nu}\left(p_{\|}\right)=\frac{4 g^{2}}{\pi}\left(g_{\|}^{\mu \nu}-\frac{p_{\|}^{\mu} p_{\|}^{\nu}}{p_{\|}^{2}}\right) .
$$

Thus after multiplying the transverse part (29), the real part of the longitudinal component ("00" component, labelled as II) of the vacuum part is simplified into

$$
\Re \Pi_{q, V}^{\|}\left(p_{0}, p_{3}\right)=-\sum_{f} \frac{\left|q_{f} B\right| g^{2} p_{3}^{2}}{2 \pi^{2} p_{\|}^{2}} .
$$

Next the medium contribution with single distribution in eq. (31) is separated as

$$
\Pi_{q, n}^{\mu \nu}\left(p_{\|}\right)=-\frac{g^{2}}{4 \pi} \int d k_{0} d k_{3} L^{\mu \nu}\left[\frac{n_{F}\left(k_{0}\right) \delta\left(k_{\|}^{2}-m_{f}^{2}\right)}{\left(q_{\|}^{2}-m_{f}^{2}\right)}+\frac{n_{F}\left(q_{0}\right) \delta\left(q_{\|}^{2}-m_{f}^{2}\right)}{\left(k_{\|}^{2}-m_{f}^{2}\right)}\right],
$$

whose real part of "|l" component for massless flavors is calculated as

$$
\Re \Pi_{q, n}^{\|}\left(p_{0}, p_{3}\right)=-\frac{4 g^{2} p_{3}^{2}}{\pi p_{\|}^{2}}\left[\frac{T \ln (2)}{p_{3}}-1-\frac{T}{p_{3}} \ln \left(1+e^{-\frac{p_{3}}{T}}\right)\right] .
$$

Thus multiplying the transverse part (29), the contribution to the real part containing single distribution function becomes

$$
\Re \Pi_{q, n}^{\|}\left(p_{0}, p_{3}\right)=-\sum_{f} \frac{\left|q_{f} B\right| g^{2} p_{3}^{2}}{2 \pi^{2} p_{\|}^{2}}\left[\frac{T \ln (2)}{p_{3}}-1-\frac{T}{p_{3}} \ln \left(1+e^{-\frac{p_{3}}{T}}\right)\right] .
$$

The medium contribution involving product of two distribution functions in eq. (31),

$$
\Pi_{q, n^{2}}^{\mu \nu}\left(p_{\|}\right)=-\frac{i g^{2}}{2} \int d k_{0} d k_{3} L^{\mu \nu}\left[n_{F}\left(k_{0}\right) n_{F}\left(q_{0}\right) \delta\left(k_{\|}^{2}-m_{f}^{2}\right) \delta\left(q_{\|}^{2}-m_{f}^{2}\right)\right],
$$

is purely imaginary, so it does not contribute to the real-part. Therefore we have

$$
\Re \Pi_{q, n^{2}}^{\|}\left(p_{0}, p_{3}\right)=0 .
$$


So the vacuum (34) and medium $((37),(39))$ contributions superpose together to give the real part of the gluon self-energy tensor due to quark-loop contribution,

$$
\begin{aligned}
\Re \Pi_{q}^{\|}\left(p_{0}, p_{3}\right) & =\Re \Pi_{q, V}^{\|}\left(p_{0}, p_{3}\right)+\Re \Pi_{q, n}^{\|}\left(p_{0}, p_{3}\right)+\Re \Pi_{q, n^{2}}^{\|}\left(p_{0}, p_{3}\right) \\
& =-\frac{g^{2}\left|q_{f} B\right| p_{3}}{2 \pi^{2} p_{\|}^{2}}\left[T \ln (2)-T \ln \left(1+e^{-\frac{p_{3}}{T}}\right)\right],
\end{aligned}
$$

which in the hard thermal loop approximation (external momentum, $p_{3}<T$ ), becomes temperature independent with the following form,

$$
\Re \Pi_{\|}^{q}\left(p_{0}, p_{3}\right)=-\sum_{f} \frac{g^{2}\left|q_{f} B\right| p_{3}^{2}}{4 \pi^{2} p_{\|}^{2}} .
$$

Defining the square of the Debye mass due to the quark-loop contribution only [50-52] as

$$
m_{q, D}^{2}=\frac{g^{2}}{4 \pi^{2}} \sum_{f}\left|q_{f} B\right|,
$$

the real part of the gluon self-energy due to the quark-loop becomes

$$
\Re \Pi_{\|}^{q}\left(p_{0}, p_{3}\right)=-\frac{m_{q, D}^{2} p_{3}^{2}}{p_{\|}^{2}} .
$$

Therefore the effective gluon propagator due to the quark-loop will be obtained from

$$
\Delta_{\|}(P)=\frac{1}{\mathbf{p}^{2}+\Pi_{\|}} .
$$

Hence the quark-loop contribution to the gluon free energy is to be calculated from

$$
\mathcal{F}_{g}^{\text {quark loop }}=\frac{\left(N_{c}^{2}-1\right)}{2} \int \frac{d^{4} P}{(2 \pi)^{4}} \ln \left(\mathbf{p}^{2}+\Pi_{\|}\right) .
$$

In the hard region, momentum (squared) $\gg \Pi_{\|}$, so it is possible to expand $\mathbf{p}^{2}+\Pi_{\|}$in powers of $\Pi_{\|} / \mathbf{p}^{2}$. After taking the leading orders (up to $\mathcal{O}\left(m_{q, D}^{4}\right)$ ) into account, eq. (45) can be written as

$$
\begin{aligned}
\mathcal{F}_{g}^{\text {quark loop }} & =\frac{\left(N_{c}^{2}-1\right)}{2} \int \frac{d^{4} P}{(2 \pi)^{4}}\left[\ln \left(\mathbf{p}^{2}\right)+\frac{\Pi_{\|}\left(p_{\|}\right)}{\mathbf{p}^{2}}-\frac{\Pi_{\|}^{2}\left(p_{\|}\right)}{2 \mathbf{p}^{4}}\right] \\
& =\frac{\left(N_{c}^{2}-1\right)}{2}\left[\int \frac{d^{4} P}{(2 \pi)^{4}} \ln \left(\mathbf{p}^{2}\right)+\int \frac{d^{4} P}{(2 \pi)^{4}} \frac{\Pi_{\|}\left(p_{\|}\right)}{\mathbf{p}^{2}}-\int \frac{d^{4} P}{(2 \pi)^{4}} \frac{\Pi_{\|}^{2}\left(p_{\|}\right)}{2 \mathbf{p}^{4}}\right] .
\end{aligned}
$$

These integrals are all divergent. Using cutoff regularization method we have solved these integrals (appendix $C$ ) and found that the divergences nearly get canceled out through 
subtraction. Thus the gluon part of the free energy in this momentum region will not contribute to the total free energy. This fact also supports the claim in [46] that in the region where momentum (squared) $\gg \Pi_{\|}$, all the self-energy corrections are negligible. However in the soft momentum region, $\Pi_{\|}=m_{q, D}^{2}$, so eq. (45) turns out to be

$$
\mathcal{F}_{g}^{\text {quark loop }}=\frac{\left(N_{c}^{2}-1\right) T}{2} \int \frac{d^{3} \mathbf{p}}{(2 \pi)^{3}} \ln \left(\mathbf{p}^{2}+m_{q, D}^{2}\right) .
$$

After solving we obtain the final value of the first part of the free energy as

$$
\mathcal{F}_{g}^{\text {quark loop }}=-\frac{\left(N_{c}^{2}-1\right) T m_{q, D}^{3}}{12 \pi} .
$$

\subsubsection{Gluon and ghost-loop contribution}

Now the structure factors, $\Pi_{T}$ and $\Pi_{L}$ are calculated using HTL approximation with temperature as the hard scale for gluon-loop momenta. Thus, in this case the effective gluon propagators for the transverse and longitudinal modes are obtained [53] as

$$
\begin{aligned}
& \Delta_{T}(P)=\frac{-1}{P^{2}+\Pi_{T}}, \\
& \Delta_{L}(P)=\frac{1}{\mathbf{p}^{2}+\Pi_{L}},
\end{aligned}
$$

respectively. So the free energy due to the transverse and longitudinal components of the gluon propagator is calculated [53] as

$$
\mathcal{F}_{g}^{\text {gluon loops }}=\left(N_{c}^{2}-1\right)\left[\int \frac{d^{4} P}{(2 \pi)^{4}} \ln \left(P^{2}+\Pi_{T}\right)+\frac{1}{2} \int \frac{d^{4} P}{(2 \pi)^{4}} \ln \left(\mathbf{p}^{2}+\Pi_{L}\right)\right],
$$

where $\Pi_{T}$ and $\Pi_{L}$ are the components of gluon self-energy calculated from the gluon and ghost loops together using the hard thermal loop perturbation theory,

$$
\begin{aligned}
& \Pi_{T}=\frac{m_{g, D}^{\prime 2}}{2} \frac{p_{0}^{2}}{\mathbf{p}^{2}}+\frac{m_{g, D}^{\prime 2}}{4} \frac{p_{0}}{\mathbf{p}}\left(1-\frac{p_{0}^{2}}{\mathbf{p}^{2}}\right) \ln \left(\frac{p_{0}+\mathbf{p}}{p_{0}-\mathbf{p}}\right) \\
& \Pi_{L}=m_{g, D}^{\prime 2}-\frac{m_{g, D}^{\prime 2}}{2} \frac{p_{0}}{\mathbf{p}} \ln \left(\frac{p_{0}+\mathbf{p}}{p_{0}-\mathbf{p}}\right)
\end{aligned}
$$

where the square of the Debye mass due to the gluon and ghost-loop is given by

$$
m_{g, D}^{\prime 2}=\frac{g^{\prime 2} T^{2} N_{c}}{3},
$$

where the QCD coupling, $g^{\prime}\left(g^{\prime 2}=4 \pi \alpha_{s}^{\prime}(T)\right)$, runs with the temperature. 
Thus, the free energy due to the gluon and ghost (51), up to $\mathcal{O}\left(m_{g, D}^{\prime 4}\right)$ can be obtained [53] as

$$
\begin{aligned}
& \mathcal{F}_{g}^{\text {gluon loops }}=-\left(N_{c}^{2}-1\right)\left[\frac{\pi^{2} T^{4}}{45}-\frac{T^{2} m_{g, D}^{\prime 2}}{24}+\frac{T m_{g, D}^{\prime 3}}{12 \pi}\right. \\
& \left.+\frac{m_{g, D}^{\prime 4}}{64 \pi^{2}}\left\{\ln \left(\frac{\Lambda}{4 \pi T}\right)-\frac{7}{2}+\gamma_{E}+\frac{\pi^{2}}{3}\right\}\right],
\end{aligned}
$$

where the renormalization scale $(\Lambda)$ is set at $2 \pi T$.

Therefore the quark (48) and gluon-loop (55) contributions together give the gluonic contribution to the free energy,

$$
\begin{aligned}
\mathcal{F}_{g}=-\left(N_{c}^{2}-1\right) \frac{T m_{q, D}^{3}}{12 \pi}-\left(N_{c}^{2}-1\right) & {\left[\frac{\pi^{2} T^{4}}{45}-\frac{T^{2} m_{g, D}^{\prime 2}}{24}+\frac{T m_{g, D}^{\prime 3}}{12 \pi}\right.} \\
+ & \left.\frac{m_{g, D}^{\prime 4}}{64 \pi^{2}}\left\{\ln \left(\frac{\Lambda}{4 \pi T}\right)-\frac{7}{2}+\gamma_{E}+\frac{\pi^{2}}{3}\right\}\right],
\end{aligned}
$$

which along with the quark contribution (19) give the free energy of hot QCD matter in the presence of a strong magnetic field,

$$
\begin{gathered}
\mathcal{F}=-\frac{N_{c} N_{f}\left|q_{f} B\right|}{4}\left[\frac{T^{2}}{3}+\int_{0}^{\left|q_{f} B\right|} \frac{d p_{\|}^{2}}{4 \pi^{2}} \ln \left[\left\{(1-2 \eta)^{2}-\frac{1}{p_{\|}^{2}}\left[m_{f}+2 \Gamma+\xi\right.\right.\right.\right. \\
\left.\left.\left.\left.+\lambda \ln \left(\frac{\left|q_{f} B\right|}{p_{\|}^{2}+m_{f}^{2}}\right)\right]^{2}\right\}\left\{1-\frac{1}{p_{\|}^{2}}\left[m_{f}+\xi+\lambda \ln \left(\frac{\left|q_{f} B\right|}{p_{\|}^{2}+m_{f}^{2}}\right)\right]^{2}\right\}\right]\right] \\
-\left(N_{c}^{2}-1\right)\left[\frac{\pi^{2} T^{4}}{45}-\frac{T^{2} m_{g, D}^{\prime 2}}{24}+\frac{T m_{g, D}^{\prime 3}}{12 \pi}+\frac{T m_{q, D}^{3}}{12 \pi}\right. \\
\left.+\frac{m_{g, D}^{\prime 4}}{64 \pi^{2}}\left\{\ln \left(\frac{\Lambda}{4 \pi T}\right)-\frac{7}{2}+\gamma_{E}+\frac{\pi^{2}}{3}\right\}\right] .
\end{gathered}
$$

\subsection{Thermodynamic properties}

In the thermodynamic limit, the negative of the free energy gives the pressure $(P=-\mathcal{F})$ for a hot QCD medium, which is found to increase in the strong magnetic field regime [6], compared to the medium in the absence of magnetic field. However, the strong magnetic field restricts the dynamics of quarks to two dimensions, which causes a reduction of the phase space and results in the decrease of both entropy and energy densities. Finally we can obtain the equation of state of the hot QCD medium from the relation between the 
energy density and pressure,

$$
P=c_{s}^{2} \varepsilon
$$

by calculating the (square) speed of sound, $c_{s}^{2}$ from the free energy in the presence of a strong magnetic field (57),

$$
c_{s}^{2}=\frac{\partial P}{\partial \varepsilon}=\frac{\partial P / \partial T}{\partial \varepsilon / \partial T}
$$

which becomes a function of both temperature and magnetic field. To see the effects of the interplay between the temperature and (strong) magnetic field quantitatively, we have computed the (square) speed of sound $\left(c_{s}^{2}\right)$ as a function of magnetic filed at different temperatures and vice versa in figures $1 \mathrm{a}$ and $1 \mathrm{~b}$, respectively and found an enhancement due to the presence of strong magnetic field. The above crucial observations on the thermodynamic observables as well as the equation of state may leave imprints on the magnetic properties of the medium and its subsequent hydrodynamic expansion in the ultrarelativistic heavy ion collisions.

\section{Magnetic properties in a strong magnetic field}

This section is devoted to explore the magnetic properties of the hot QCD matter in the presence of strong magnetic field. For this purpose we are going to calculate the lowest and the next-to-lowest order responses of the medium to the magnetic field, i.e. the magnetization and the magnetic susceptibility in the next subsections, respectively.

\subsection{Magnetization}

Once the free energy $(\mathcal{F})$ due to a medium of interacting quarks and gluons is known, the magnetization $(\mathcal{M})$ can be obtained from the following definition,

$$
\mathcal{M}=-\frac{\partial \mathcal{F}}{\partial(e B)}
$$




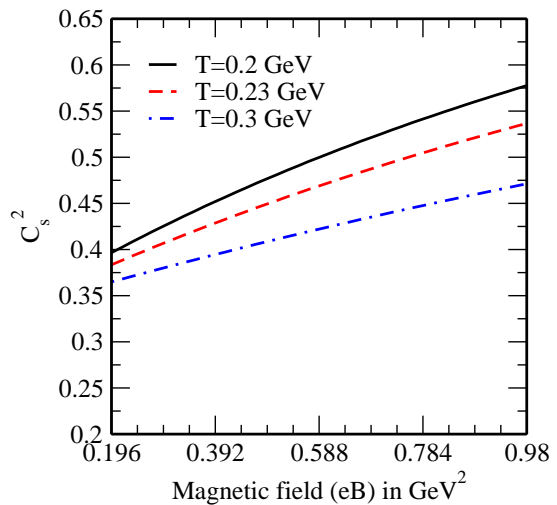

a

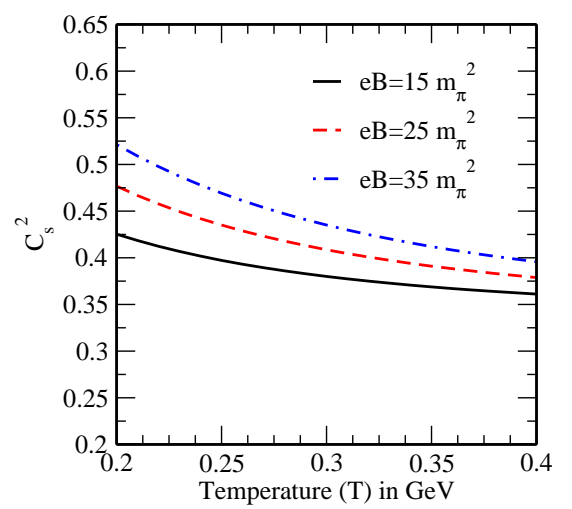

$\mathrm{b}$

Figure 1: The variation of square of the speed of sound with strong magnetic field at fixed temperatures (a) and the variation with temperature in the presence of strong magnetic fields (b).

The final expression of $\mathcal{M}$ is given by

$$
\begin{aligned}
\mathcal{M}= & \frac{N_{c} N_{f}}{4}\left[\frac{T^{2}}{3}+\int_{0}^{\left|q_{f} B\right|} \frac{d p_{\|}^{2}}{4 \pi^{2}} \ln \left[\left\{(1-2 \eta)^{2}-\frac{1}{p_{\|}^{2}}\left[m_{f}+2 \Gamma+\xi\right.\right.\right.\right. \\
& \left.\left.\left.+\lambda \ln \left(\frac{\left|q_{f} B\right|}{p_{\|}^{2}+m_{f}^{2}}\right)\right]^{2}\right\}\left\{1-\frac{1}{p_{\|}^{2}}\left[m_{f}+\xi+\lambda \ln \left(\frac{\left|q_{f} B\right|}{p_{\|}^{2}+m_{f}^{2}}\right)\right]^{2}\right\}\right] \\
& +\frac{N_{c} N_{f}\left|q_{f} B\right|}{16 \pi^{2}} \int_{0}^{\left|q_{f} B\right|} d p_{\|}^{2}\left[\frac{1}{(1-2 \eta)^{2}-\frac{1}{p_{\|}^{2}}\left\{m_{f}+2 \Gamma+\xi+\lambda \ln \left(\frac{\left|q_{f} B\right|}{p_{\|}^{2}+m_{f}^{2}}\right)\right\}^{2}}\right. \\
& \times\left[\frac{g^{2}(1-2 \eta)}{3 \pi^{2} m_{f}^{2}} \ln \left(\frac{\left|q_{f} B\right|}{m_{f}^{2}}\right)-\frac{2}{p_{\|}^{2}}\left\{m_{f}+2 \Gamma+\xi+\lambda \ln \left(\frac{\left|q_{f} B\right|}{p_{\|}^{2}+m_{f}^{2}}\right)\right\}\right. \\
& \left.\times\left\{\frac{g^{2}}{3 \pi^{2} m_{f}} \ln \left(\frac{\left|q_{f} B\right|}{m_{f}^{2}}\right)+\frac{\lambda}{\left|q_{f} B\right|}\right\}\right]-\frac{1}{1-\frac{1}{p_{\|}^{2}}\left\{m_{f}+\xi+\lambda \ln \left(\frac{\left|q_{f} B\right|}{p_{\|}^{2}+m_{f}^{2}}\right)\right\}^{2}} \\
& \left.\times \frac{2 \lambda}{p_{\|}^{2}\left|q_{f} B\right|}\left\{m_{f}+\xi+\lambda \ln \left(\frac{\left|q_{f} B\right|}{p_{\|}^{2}+m_{f}^{2}}\right)\right\}\right]+\frac{\left(N_{c}^{2}-1\right) g^{2} T m_{q, D}}{32 \pi^{3}} .
\end{aligned}
$$

To analyse the magnetic behavior of QCD matter in a strong magnetic field, we have plotted the magnetization as a function of magnetic field at fixed temperatures $0.2 \mathrm{GeV}$, $0.23 \mathrm{GeV}$ and $0.3 \mathrm{GeV}$ in figure $2 \mathrm{a}$ and vice versa at magnetic fields, $15 \mathrm{~m}_{\pi}^{2}, 25 \mathrm{~m}_{\pi}^{2}$ and 


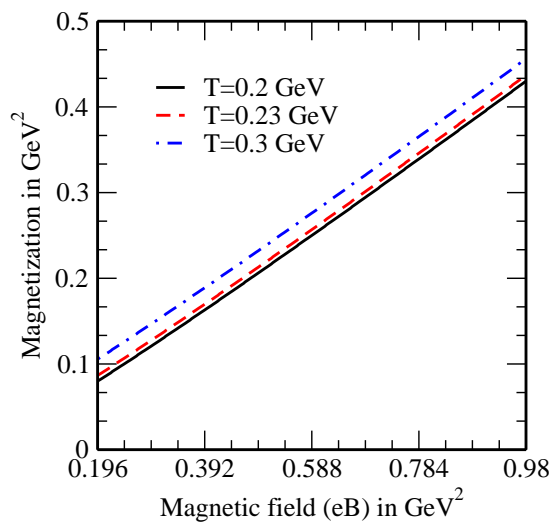

a

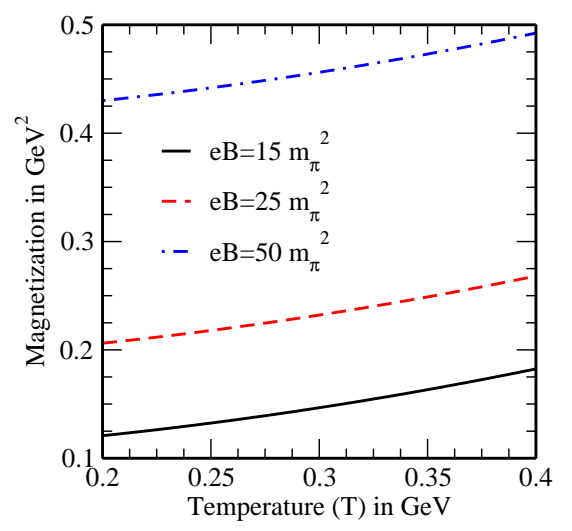

$\mathrm{b}$

Figure 2: The variation of magnetization as a function of the magnetic field at different temperatures (a) and as a function of the temperature at different magnetic fields (b).

$50 m_{\pi}^{2}$ in figure $2 \mathrm{~b}$. Similarly the magnetic susceptibility is also plotted as a function of temperature at different strong magnetic fields. In all these plots, we have set the ranges of magnetic field and temperature compatible to the strong magnetic field limit $\left(e B \gg T^{2}\right)$. We have observed that in the strong magnetic field limit, the magnetization increases almost linearly with the magnetic field and the increase is relatively larger for a hotter medium (in figure 2a). On the other hand the magnetization increases with the temperature too but the increase is meagre (in figure 2b). The above (positive) magnetization can thus be explained by the decrease of free energy of the hot QCD matter in an ambient strong magnetic field, so it behaves as a paramagnetic medium. Minimization of the free energy induces a force, $F \equiv-\nabla \mathcal{F}$ which might affect the elliptic flow and causes a change in the shape of the QCD matter in the transverse plane of the noncentral heavy ion collision, known as paramagnetic squeezing. Recent calculations in lattice [54-56] and in hadron resonance gas model [57] also indicate that a hot QCD medium in a strong magnetic field is a paramagnetic one.

To see how the interactions among quarks and gluons affect the magnetization, we have computed the magnetization in units of its noninteracting value as a function of magnetic field at fixed temperatures in figure $3 \mathrm{a}$ and vice versa in figure $3 \mathrm{~b}$. We have observed that the magnetization gets enhanced due to the interaction and the enhancement is more pronounced with the increase of magnetic field. However the interaction has the opposite effect on the variation of magnetization with the temperature where the magnetization decreases with the temperature. This is due to the fact that as the temperature 


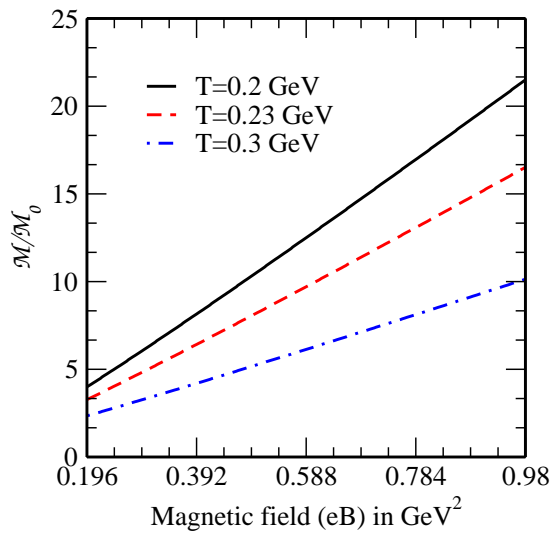

a

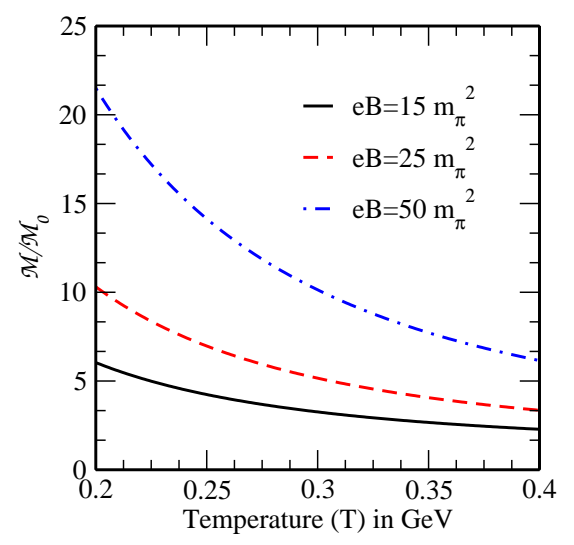

$\mathrm{b}$

Figure 3: Variation of magnetization normalized by its ideal value as a function of the magnetic field at different temperatures (a) and as a function of the temperature at different magnetic fields (b).

increases, the orientation of magnetic dipoles becomes more randomized, as a result the magnetization of the interacting quarks approaches beyond a temperature (greater than the magnetic interaction energy) to its value attributed by the seemingly noninteracting quarks.

\subsection{Magnetic susceptibility}

The magnetic susceptibility $(\chi)$ is a higher-order cumulant of free energy, which is obtained from the free energy as

$$
\chi=-\frac{\partial^{2} \mathcal{F}}{\partial(e B)^{2}} .
$$


The final expression of $\chi$ is given by

$$
\begin{aligned}
& \chi=\frac{N_{c} N_{f}}{8 \pi^{2}} \int_{0}^{\left|q_{f} B\right|} d p_{\|}^{2}\left[\frac{1}{(1-2 \eta)^{2}-\frac{1}{p_{\|}^{2}}\left\{m_{f}+2 \Gamma+\xi+\lambda \ln \left(\frac{\left|q_{f} B\right|}{p_{\|}^{2}+m_{f}^{2}}\right)\right\}^{2}}\right. \\
& \times\left[\frac{g^{2}(1-2 \eta)}{3 \pi^{2} m_{f}^{2}} \ln \left(\frac{\left|q_{f} B\right|}{m_{f}^{2}}\right)-\frac{2}{p_{\|}^{2}}\left\{m_{f}+2 \Gamma+\xi+\lambda \ln \left(\frac{\left|q_{f} B\right|}{p_{\|}^{2}+m_{f}^{2}}\right)\right\}\right. \\
& \left.\times\left\{\frac{g^{2}}{3 \pi^{2} m_{f}} \ln \left(\frac{\left|q_{f} B\right|}{m_{f}^{2}}\right)+\frac{\lambda}{\left|q_{f} B\right|}\right\}\right]-\frac{1}{1-\frac{1}{p_{\|}^{2}}\left\{m_{f}+\xi+\lambda \ln \left(\frac{\left|q_{f} B\right|}{p_{\|}^{2}+m_{f}^{2}}\right)\right\}^{2}} \\
& \left.\times \frac{2 \lambda}{p_{\|}^{2}\left|q_{f} B\right|}\left\{m_{f}+\xi+\lambda \ln \left(\frac{\left|q_{f} B\right|}{p_{\|}^{2}+m_{f}^{2}}\right)\right\}\right] \\
& +\frac{N_{c} N_{f}\left|q_{f} B\right|}{16 \pi^{2}} \int_{0}^{\left|q_{f} B\right|} d p_{\|}^{2}\left[\frac{1}{(1-2 \eta)^{2}-\frac{1}{p_{\|}^{2}}\left\{m_{f}+2 \Gamma+\xi+\lambda \ln \left(\frac{\left|q_{f} B\right|}{p_{\|}^{2}+m_{f}^{2}}\right)\right\}^{2}}\right. \\
& \times\left[\frac{1}{2}\left\{\frac{g^{2}}{3 \pi^{2} m_{f}^{2}} \ln \left(\frac{\left|q_{f} B\right|}{m_{f}^{2}}\right)\right\}^{2}+\frac{g^{2}(1-2 \eta)}{3 \pi^{2} m_{f}^{2}\left|q_{f} B\right|}-\frac{2}{p_{\|}^{2}}\left\{\frac{g^{2}}{3 \pi^{2} m_{f}\left|q_{f} B\right|}-\frac{\lambda}{\left|q_{f} B\right|^{2}}\right\}\right. \\
& \left.\times\left\{m_{f}+2 \Gamma+\xi+\lambda \ln \left(\frac{\left|q_{f} B\right|}{p_{\|}^{2}+m_{f}^{2}}\right)\right\}-\frac{2}{p_{\|}^{2}}\left\{\frac{g^{2}}{3 \pi^{2} m_{f}} \ln \left(\frac{\left|q_{f} B\right|}{m_{f}^{2}}\right)+\frac{\lambda}{\left|q_{f} B\right|}\right\}^{2}\right] \\
& 1 \\
& \overline{\left[(1-2 \eta)^{2}-\frac{1}{p_{\|}^{2}}\left\{m_{f}+2 \Gamma+\xi+\lambda \ln \left(\frac{\left|q_{f} B\right|}{p_{\|}^{2}+m_{f}^{2}}\right)\right\}^{2}\right]^{2}} \\
& \times\left[-\frac{2}{p_{\|}^{2}}\left\{m_{f}+2 \Gamma+\xi+\lambda \ln \left(\frac{\left|q_{f} B\right|}{p_{\|}^{2}+m_{f}^{2}}\right)\right\}\left\{\frac{g^{2}}{3 \pi^{2} m_{f}} \ln \left(\frac{\left|q_{f} B\right|}{m_{f}^{2}}\right)+\frac{\lambda}{\left|q_{f} B\right|}\right\}\right. \\
& \left.+\frac{g^{2}(1-2 \eta)}{3 \pi^{2} m_{f}^{2}} \ln \left(\frac{\left|q_{f} B\right|}{m_{f}^{2}}\right)\right]^{2}+\frac{1}{\left[1-\frac{1}{p_{\|}^{2}}\left\{m_{f}+\xi+\lambda \ln \left(\frac{\left|q_{f} B\right|}{p_{\|}^{2}+m_{f}^{2}}\right)\right\}^{2}\right]^{2}} \\
& \times\left[\frac{2 \lambda}{p_{\|}^{2}\left|q_{f} B\right|^{2}}\left\{m_{f}+\xi+\lambda \ln \left(\frac{\left|q_{f} B\right|}{p_{\|}^{2}+m_{f}^{2}}\right)-\lambda\right\}-\frac{2 \lambda}{p_{\|}^{4}\left|q_{f} B\right|^{2}}\right. \\
& \left.\left.\times\left\{m_{f}+\xi+\lambda \ln \left(\frac{\left|q_{f} B\right|}{p_{\|}^{2}+m_{f}^{2}}\right)+\lambda\right\}\left\{m_{f}+\xi+\lambda \ln \left(\frac{\left|q_{f} B\right|}{p_{\|}^{2}+m_{f}^{2}}\right)\right\}^{2}\right]\right] \\
& +\frac{\left(N_{c}^{2}-1\right) g^{4} T}{256 m_{q, D} \pi^{5}} .
\end{aligned}
$$




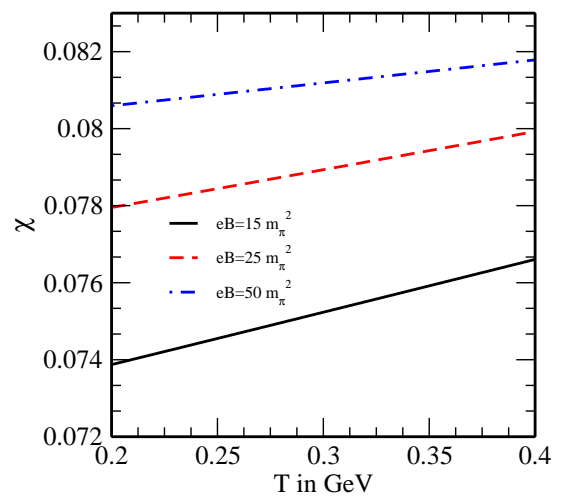

Figure 4: The variation of magnetic susceptibility as a function of temperature at different magnetic fields.

To investigate the response of magnetic field of the hot QCD medium quantitatively, we have computed the magnetic susceptibility as a function of the temperature at different strong magnetic fields in figure 4, which comes out to be positive and increases with both temperature and magnetic field albeit the increase with the temperature is meagre. Since the susceptibility remains positive therefore it reaffirms the paramagnetic nature of hot QCD medium in a strong magnetic field.

Thus the free energy and its higher-order derivatives with respect to either magnetic field or temperature give the information about the magnetic and thermodynamic properties of a given medium in its different phases, respectively. In this work we have explored the magnetic behavior of QCD matter in a temperature range $200-400 \mathrm{MeV}$ in the presence of strong magnetic fields by calculating the magnetization and magnetic susceptibility, which are, respectively, given by the first- and second-order derivatives of the free energy with respect to the magnetic field. Similarly, in a pure thermal medium, the quarknumber susceptibility can be obtained by differentiating the free energy with respect to the quark chemical potential to observe the baryon-number fluctuation, which facilitates to locate the critical point of phase transition [58, 59]. Also the chiral susceptibility, which can be determined by the second-order derivative of the free energy with respect to the quark mass, helps to characterize the chiral phase transition in QCD [60, 61]. 


\section{Bjorken expansion in the presence of a strong magnetic field}

In the vacuum the magnetic field decays very fast [8] so that it becomes tiny before a locally equilibrated thermal medium is created. Thus there may be some possible action of magnetic field on the static and dynamic properties of the medium produced in URHIC. However, some recent observations predict that the decay of magnetic field might be significantly slowed down due to the transport properties of the medium, viz. electrical conductivity $[32,62,63]$, as a result the magnetic field may be strong enough till the thermal medium is produced locally. This motivates to study the effect of strong magnetic field on the dynamics of the matter produced in the heavy ion collision.

The hydrodynamic expansion of the medium is given by the conservation of the energymomentum tensor,

$$
\partial_{\mu} T^{\mu \nu}=0
$$

where, in the absence of dissipative forces, it can be obtained as

$$
T^{\mu \nu}=(\varepsilon+P) u^{\mu} u^{\nu}-g^{\mu \nu} P,
$$

where $\varepsilon$ and $P$ are the energy density and the pressure of the medium, respectively. These are obtained from the free energy of the thermal QCD medium in a strong magnetic field, which is shown to behave as a paramagnetic medium, i.e. having positive magnetization.

Therefore the ideal fluid gives the longitudinal boost-invariant Bjorken expansion,

$$
\frac{d \varepsilon}{d \tau}=-\frac{\varepsilon+P}{\tau} .
$$

Using the equation of state $\left(P=c_{s}^{2} \varepsilon\right)$ for thermal QCD medium in a strong magnetic field, the above first-order differential equation can be solved to give the evolution of energy density as a function of the proper-time,

$$
\varepsilon(\tau) \tau^{1+c_{s}^{2}}=\varepsilon\left(\tau_{i}\right) \tau_{i}^{1+c_{s}^{2}},
$$

where $c_{s}^{2}$ is taken from the eq. (59) as a function of both temperature and magnetic field, whereas in usual hydrodynamics, $c_{s}^{2}$ is either taken as a constant $(1 / 3)$ for noninteracting equation of state or taken as a function of temperature only (for baryonless matter). It is very important to mention here that the effect of magnetic field on the expansion dynamics enters through the thermodynamic equation of state, unlike the usual magnetohydrodynamics for the static as well as dynamic magnetic fields, where the energy-momentum tensor $\left(T^{\mu \nu}\right)$ is decomposed into the matter $\left(T_{M}^{\mu \nu}\right)$ and the (external) field $\left(T_{B}^{\mu \nu}\right)$ contributions, as in $[36,37]$. The latter comes as a product of the canonically conjugate variables. 
Here these are magnetization and magnetic field for which they have taken some specific values for them. On the contrary we have started with the EoS for the hot QCD medium in the presence of a strong magnetic field, where both pressure and energy density are functions of temperature and magnetic field and thus it automatically guarantees the effect of (strong) magnetic field into the EoS, confirming the paramagnetic nature. As a result, in our case, the energy-momentum tensor (that contains the matter part) itself incorporates the effects of temperature, magnetic field and initial magnetization, so, there is no need to include the magnetic field part $\left(T_{B}^{\mu \nu}\right)$ in the energy-momentum tensor further.

The proper-time evolution of energy density can be translated, by the equation of state $\left(\varepsilon \sim T^{4}\right)$, into the evolution of temperature with (proper) time, known as cooling law. As an example, using the noninteracting equation of state for a thermal QCD medium, eq. (67) can be converted into

$$
T^{3} \tau=T_{i}^{3} \tau_{i}
$$

The corrections to the Bjorken expansion for ideal fluid can be incorporated by adding the shear stress tensor, $\pi^{\mu \nu}$ to the ideal case (65),

$$
T^{\mu \nu}=(\varepsilon+P) u^{\mu} u^{\nu}-g^{\mu \nu} P+\pi^{\mu \nu},
$$

which can be expressed in terms of the velocity-gradient,

$$
\pi^{\mu \nu}=\eta\left(\nabla^{\mu} u^{\nu}+\nabla^{\nu} u^{\mu}-\frac{2}{3} \nabla^{\mu \nu} \nabla^{\rho} u_{\rho}\right),
$$

where $\eta$ is the shear viscosity and $\nabla^{\mu}=\nabla^{\mu \nu} \partial_{\nu}$ with $\nabla^{\mu \nu}=g^{\mu \nu}-u^{\mu} u^{\nu}$.

In a first-order viscous hydrodynamics, the shear stress tensor is written in terms of the first-order symmetrized velocity gradient $\left(\left\langle\nabla^{\mu} u^{\nu}\right\rangle\right)$,

$$
\pi^{\mu \nu}=\eta\left\langle\nabla^{\mu} u^{\nu}\right\rangle
$$

whereas for a second-order viscous hydrodynamics, Israel-Stewart theory [64] modifies the equation of motion for the ideal fluid (66) into $[65,66]$,

$$
\frac{d \varepsilon}{d \tau}=-\frac{1}{\tau}(\varepsilon+P-\Phi)
$$

where the shear stress $(\Phi)$ will asymptotically be reduced into its first-order value after the relaxation time $\left(\tau_{\pi}\right)$ is elapsed. In the Navier-Stokes limit, the one-dimensional boostinvariant expansion gives [65],

$$
\Phi=\frac{4 \eta}{3 \tau} .
$$




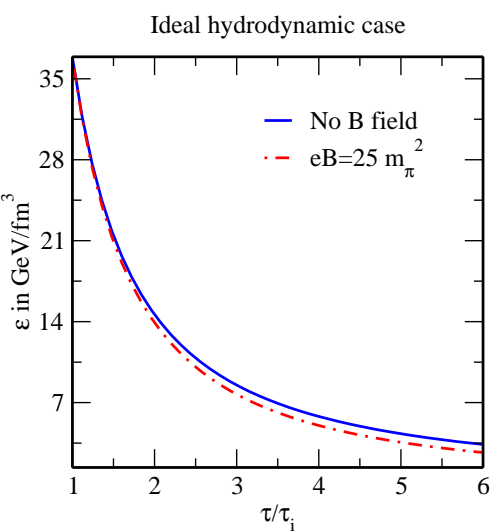

a

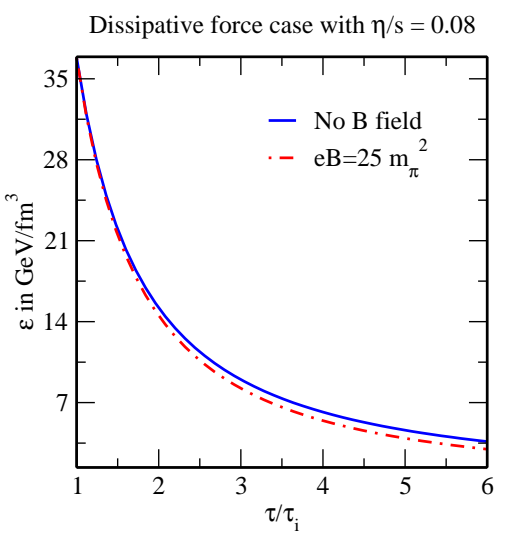

$\mathrm{b}$

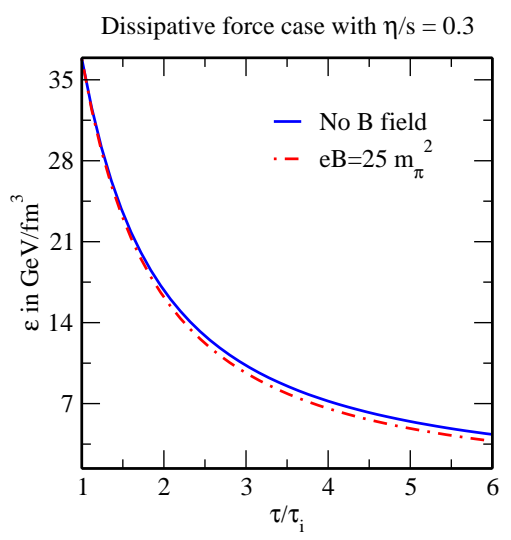

c

Figure 5: Evolution of the energy density in case of (a) ideal hydrodynamics and in the presence of dissipative forces with the ratio, (b) $\frac{\eta}{s}=0.08$ and (c) $\frac{\eta}{s}=0.3$, respectively.

Thus substituting the value of $\Phi$ in eq. (72), the Bjorken longitudinal expansion for dissipative fluid can be obtained as

$$
\frac{d \varepsilon}{d \tau}+\frac{\varepsilon+P}{\tau}=\frac{4 \eta}{3 \tau^{2}}
$$

The solution of eq. (74) with the $\operatorname{EoS} P=c_{s}^{2} \varepsilon$, is determined as

$$
\varepsilon(\tau) \tau^{1+c_{s}^{2}}+\left\{\frac{4 a_{f} T_{i}^{3} \tau_{i}}{3}\right\}\left\{\frac{\eta}{s}\right\} \frac{\tau^{1+c_{s}^{2}}}{\tilde{\tau}^{2}}=\varepsilon\left(\tau_{i}\right) \tau_{i}^{1+c_{s}^{2}}+\left\{\frac{4 a_{f} T_{i}^{3} \tau_{i}}{3}\right\}\left\{\frac{\eta}{s}\right\} \frac{\tau_{i}^{1+c_{s}^{2}}}{{\tilde{\tau_{i}}}^{2}}
$$

where $a_{f}=\left(16+21 N_{f} / 2\right) \pi^{2} / 90, \tilde{\tau}^{2}$ and $\tilde{\tau}_{i}^{2}$ denote $\left(1-c_{s}^{2}\right) \tau^{2}$ and $\left(1-c_{s}^{2}\right) \tau_{i}^{2}$, respectively. In the above solution, the first and the second terms in both sides represent the contributions due to the zeroth-order expansion and due to the viscous corrections, respectively. In the present work we have used the shear viscosity to entropy ratio, $\eta / s$ as 0.08 from the AdS/CFT [67] calculation and 0.3 from the perturbative QCD [68] calculation. In our calculations we have also set $\tau_{i}=0.25 \mathrm{fm} / \mathrm{c}, T_{i}=0.39 \mathrm{GeV}$ for centrality $(40-50) \%$ at energy $\sqrt{s_{N N}}=200 \mathrm{GeV}$ from the RHIC Au+Au data [69], and through the equation of state the initial temperature gives $\varepsilon_{i}=36.833 \mathrm{GeV} / \mathrm{fm}^{3}$.

Similar to the cooling law (68) in ideal hydrodynamics, the cooling law in a first-order dissipative hydrodynamics can also be obtained in terms of the $\eta / s$ ratio,

$$
T(\tau)=T_{i}\left(\frac{\tau_{i}}{\tau}\right)^{1 / 3}\left[1+\frac{2 \eta}{3 \tau_{i} T_{i} s}\left\{1-\left(\frac{\tau_{i}}{\tau}\right)^{2 / 3}\right\}\right]
$$


To know how an ambient strong magnetic field could affect the hydrodynamic expansion quantitatively, we have observed the evolution of the energy density $(\varepsilon)$ of a hot QCD medium with the proper-time $(\tau)$ in the absence (solid lines) and in the presence of strong magnetic field (dotted-dashed lines) in figures $5 \mathrm{a}$ and $5 \mathrm{~b}$ and $5 \mathrm{c}$ for the ideal and dissipative fluids, respectively. The energy density always decreases with the proper time, but in the presence of strong magnetic field, the decrease of $\varepsilon$ with $\tau$ becomes faster as compared to the case in the absence of magnetic field. This observation can be envisaged from our earlier observation on the equation of state (figure 1), where the speed of sound gets enhanced due to the presence of strong magnetic field. Similar behavior in the energy density as a function of proper time is also noticed in the presence of dissipative forces, i.e. with the nonzero shear viscosity to entropy ratio $(\eta / s)$.

\section{Conclusions}

We have studied the response of strong magnetic field on a hot QCD medium by estimating the magnetization and the magnetic susceptibility from its one-loop free energy evaluated in the strong magnetic field limit $\left(e B \gg T^{2}\right)$, which may be realized in the non-central events of ultra-relativistic heavy ion collisions. Both the magnetization and magnetic susceptibility have been emanated from the quark and gluon contributions through their respective one-loop self-energies, where the quark contribution is largely affected by the magnetic field unlike the gluon contribution. The positive values of both magnetization and magnetic susceptibility, which in turn increase with the magnetic field, have affirmed the paramagnetic nature of the hot QCD medium in the presence of a strong magnetic field. In addition, we have explored the effect of strong magnetic field on the evolution of the QCD medium both in the presence and absence of dissipative forces, where the evolution of the energy density with proper time is observed to get faster than that in a pure thermal medium in the absence of magnetic field.

\section{Acknowledgment}

We would like to thank Prof. Somendra Mohan Bhattacharjee for fruitful discussions during the preparation of this manuscript. 


\section{Appendices}

\section{A Quark self-energy in a strong magnetic field}

In the presence of a magnetic field, the motion of a quark perpendicular to the direction of magnetic field is quantized in terms of Landau levels. In the SMF limit, i.e. in the LLL approximation $k_{\perp}^{2} \ll\left|q_{f} B\right|$ and this leads $\exp \left(-k_{\perp}^{2} /\left|q_{f} B\right|\right) \approx 1$. Also the upper limits of the momentum integrals are set at $\left|q_{f} B\right|$ and $p_{\perp} \approx 0$ [42], so we have

$$
(P-K)^{2} \simeq\left(p_{\|}-k_{\|}\right)^{2}-k_{\perp}^{2} .
$$

Therefore the quark self-energy (16) becomes a function of the longitudinal component of momentum only with the following form,

$$
\begin{aligned}
\Sigma\left(p_{\|}\right)= & \frac{-8 g^{2} i}{3(2 \pi)^{4}} \int d^{2} k_{\perp} d^{2} k_{\|}\left[J_{+}\left(\gamma^{0} k_{0}-\gamma^{3} k_{3}\right)-2 m_{f}\right] \\
& \times\left[\frac{1}{k_{\|}^{2}-m_{f}^{2}+i \epsilon}+2 \pi i n_{F}\left(k_{0}\right) \delta\left(k_{\|}^{2}-m_{f}^{2}\right)\right] \\
& \times\left[\frac{1}{\left(p_{\|}-k_{\|}\right)^{2}-k_{\perp}^{2}+i \epsilon}-2 \pi i n_{B}\left(p_{0}-k_{0}\right) \delta\left(\left(p_{\|}-k_{\|}\right)^{2}-k_{\perp}^{2}\right)\right] \\
\equiv & \Sigma_{V}\left(p_{\|}\right)+\Sigma_{n}\left(p_{\|}\right)+\Sigma_{n^{2}}\left(p_{\|}\right),
\end{aligned}
$$

where $\Sigma_{V}\left(p_{\|}\right), \Sigma_{n}\left(p_{\|}\right)$and $\Sigma_{n^{2}}\left(p_{\|}\right)$denote the vacuum part, medium parts containing single distribution function and product of two distribution functions, respectively.

\section{A.1 Vacuum part}

The vacuum part of the quark self-energy from eq. (A.78) can be written as

$$
\begin{aligned}
\Sigma_{V}\left(p_{\|}\right)= & \frac{-8 g^{2} i}{3(2 \pi)^{4}} \int d^{2} k_{\perp} d^{2} k_{\|}\left[J_{+}\left(\gamma^{0} k_{0}-\gamma^{3} k_{3}\right)-2 m_{f}\right] \\
& \times\left[\frac{1}{k_{\|}^{2}-m_{f}^{2}+i \epsilon}\right]\left[\frac{1}{\left(p_{\|}-k_{\|}\right)^{2}-k_{\perp}^{2}+i \epsilon}\right] .
\end{aligned}
$$

Using the identity,

$$
\frac{1}{x \pm y \pm i \epsilon}=\mathrm{P}\left(\frac{1}{x \pm y}\right) \mp i \pi \delta(x \pm y)
$$


we obtain the real part with the following form,

$$
\begin{aligned}
\Sigma_{V}\left(p_{\|}\right) & =\frac{-8 g^{2}}{3(2 \pi)^{4}} \int d^{2} k_{\perp} \int d^{2} k_{\|}\left[J_{+} \gamma^{\|} \cdot k_{\|}-2 m_{f}\right] \frac{i}{\left[k_{\|}^{2}-m_{f}^{2}\right]\left[\left(p_{\|}-k_{\|}\right)^{2}-k_{\perp}^{2}\right]} \\
& \equiv \frac{-8 g^{2}}{3(2 \pi)^{4}} \int d^{2} k_{\perp} I .
\end{aligned}
$$

Using Feynman parametrization method and Wick rotation, integration $I$ can be solved with the change of variable from $k_{\|}$to $k^{\prime}$ through the replacement $k_{\|}-z p_{\|}=k^{\prime}$,

$$
\begin{aligned}
I & =-\int_{0}^{1} d z d^{2} k^{\prime} \frac{z J_{+} \gamma^{\|} \cdot p_{\|}-2 m_{f}}{\left[k^{\prime 2}-z(1-z) p_{\|}^{2}+z k_{\perp}^{2}+(1-z) m_{f}^{2}\right]^{2}} \\
& =\frac{\pi}{\left|q_{f} B\right|} \int_{0}^{1} d z\left(z J_{+} \gamma^{\|} \cdot p_{\|}-2 m_{f}\right)+\pi \int_{0}^{1} d z \frac{-z J_{+} \gamma^{\|} \cdot p_{\|}+2 m_{f}}{-z(1-z) p_{\|}^{2}+z k_{\perp}^{2}+(1-z) m_{f}^{2}} \\
& \equiv I^{a}+I^{b}
\end{aligned}
$$

After solving the integral $I^{a}$ analytically, we get

$$
I^{a}=\frac{\pi}{2\left|q_{f} B\right|}\left(J_{+} \gamma^{\|} \cdot p_{\|}-4 m_{f}\right) .
$$

The integral $I^{b}$ is solved by expanding it in a Taylor series around the mass-shell condition $-\gamma^{\|} \cdot p_{\|}=m_{f}$ in a strong magnetic field,

$$
I^{b}=A+B\left(\gamma^{\|} \cdot p_{\|}-m_{f}\right)+C\left(\gamma^{\|} \cdot p_{\|}-m_{f}\right)^{2}+\cdots .
$$

Up to first order (dropping the higher-order terms), $I^{b}$ is given by

$$
I^{b}=A+B\left(\gamma^{\|} \cdot p_{\|}-m_{f}\right),
$$

where the coefficients, $A$ and $B$ are calculated as follows,

$$
\begin{aligned}
A & =\left.I^{b}\right|_{\gamma^{\|} \cdot p_{\|}=m_{f}} \\
& =-\pi m_{f} \int_{0}^{1} d z \frac{z J_{+}-2}{m_{f}^{2}(1-z)^{2}+z k_{\perp}^{2}}, \\
B & =\left.\frac{\partial I^{b}}{\partial\left(\gamma^{\|} \cdot p_{\|}\right)}\right|_{\gamma^{\|} \cdot p_{\|}=m_{f}} \\
& =-\pi \int_{0}^{1} d z \frac{z J_{+}}{(1-z)^{2} m_{f}^{2}+z k_{\perp}^{2}}-\pi \int_{0}^{1} d z \frac{2 m_{f}^{2} z(1-z)\left(z J_{+}-2\right)}{\left\{(1-z)^{2} m_{f}^{2}+z k_{\perp}^{2}\right\}^{2}} .
\end{aligned}
$$


The Feynman integrations in equations (A.86) and (A.87) yield the expressions for the coefficients $A$ and $B$ which for masses of two light flavors $u$ and $d$, are simplified into,

$$
\begin{aligned}
A & \simeq-\frac{\pi J_{+}}{2 m_{f}} \ln \left(\frac{k_{\perp}^{2}}{m_{f}^{2}}\right), \\
B & \simeq \frac{\pi J_{+}}{2 m_{f}^{2}} \ln \left(\frac{k_{\perp}^{2}}{m_{f}^{2}}\right) .
\end{aligned}
$$

Therefore the integral, $I^{b}$ takes the following form,

$$
I^{b}=-\frac{\pi J_{+}}{m_{f}} \ln \left(\frac{k_{\perp}^{2}}{m_{f}^{2}}\right)+\frac{\pi J_{+}}{2 m_{f}^{2}}\left(\gamma^{\|} \cdot p_{\|}\right) \ln \left(\frac{k_{\perp}^{2}}{m_{f}^{2}}\right) .
$$

Now the $k_{\|}$-integration ( $I$ ) in eq. (A.82) turns out to be

$$
I=\frac{\pi}{2\left|q_{f} B\right|}\left(J_{+} \gamma^{\|} \cdot p_{\|}-4 m_{f}\right)-\frac{\pi J_{+}}{m_{f}} \ln \left(\frac{k_{\perp}^{2}}{m_{f}^{2}}\right)+\frac{\pi J_{+}}{2 m_{f}^{2}}\left(\gamma^{\|} \cdot p_{\|}\right) \ln \left(\frac{k_{\perp}^{2}}{m_{f}^{2}}\right) .
$$

Thus the real part of the vacuum contribution in eq. (A.81) becomes

$$
\begin{aligned}
\Sigma_{V}\left(p_{\|}\right)= & \frac{-8 g^{2} \pi}{3(2 \pi)^{4}} \int_{0}^{\left|q_{f} B\right|} d k_{\perp}^{2} I \\
= & \frac{J_{+}\left(\gamma^{\|} \cdot p_{\|}\right) g^{2}}{12 \pi^{2}}\left[-1-\frac{\left|q_{f} B\right|}{m_{f}^{2}}\left\{\ln \left(\frac{\left|q_{f} B\right|}{m_{f}^{2}}\right)-1\right\}\right] \\
& +\frac{g^{2} m_{f}}{3 \pi^{2}}\left[1+\frac{J_{+}\left|q_{f} B\right|}{2 m_{f}^{2}}\left\{\ln \left(\frac{\left|q_{f} B\right|}{m_{f}^{2}}\right)-1\right\}\right] .
\end{aligned}
$$

\section{A.2 Medium part}

The medium part, $\Sigma_{n}\left(p_{\|}\right)$, which depends on the quark and gluon distribution functions linearly is given by

$$
\begin{aligned}
\Sigma_{n}\left(p_{\|}\right)= & \frac{8 g^{2}}{3(2 \pi)^{3}} \int d^{2} k_{\perp} d k_{3} d k_{0}\left[J_{+}\left(\gamma^{0} k_{0}-\gamma^{3} k_{3}\right)-2 m_{f}\right] \\
& \times\left[\frac{\delta\left(k_{\|}^{2}-m_{f}^{2}\right) n_{F}\left(k_{0}\right)}{\left(p_{\|}-k_{\|}\right)^{2}-k_{\perp}^{2}}+\frac{\delta\left(\left(p_{\|}-k_{\|}\right)^{2}-k_{\perp}^{2}\right)\left[-n_{B}\left(p_{0}-k_{0}\right)\right]}{k_{\|}^{2}-m_{f}^{2}}\right] \\
\equiv & \Sigma_{n_{F}}\left(p_{\|}\right)+\Sigma_{n_{B}}\left(p_{\|}\right) .
\end{aligned}
$$


The part containing only the quark distribution function is rewritten as

$$
\Sigma_{n_{F}}\left(p_{\|}\right)=\frac{8 g^{2}}{3(2 \pi)^{3}} \int d^{2} k_{\perp} d k_{3} d k_{0}\left[J_{+}\left(\gamma^{0} k_{0}-\gamma^{3} k_{3}\right)-2 m_{f}\right] \frac{\delta\left(k_{0}^{2}-\omega_{k}^{2}\right) n_{F}\left(k_{0}\right)}{\left(p_{\|}-k_{\|}\right)^{2}-k_{\perp}^{2}},
$$

where $\omega_{k}^{2}=k_{3}^{2}+m_{f}^{2}$. Using the property of Dirac delta function and considering the highest order of $p_{\|}^{2}$ as $\left|q_{f} B\right|$ in the strong magnetic field limit $\left(e B \gg T^{2}\right)$ [42], integration over $k_{0}$ is done. So, we are left with $k_{3}$ and $k_{\perp}$ integrations,

$$
\Sigma_{n_{F}}\left(p_{\|}\right)=\frac{-8 g^{2}}{3(2 \pi)^{3}} \int d k_{3} \frac{n_{F}\left(\omega_{k}\right)}{\omega_{k}}\left(J_{+} \gamma^{3} k_{3}+2 m_{f}\right) \int d^{2} k_{\perp} \frac{1}{p_{\|}^{2}+m_{f}^{2}-k_{\perp}^{2}},
$$

where the $k_{3}$-integration is performed [70] to obtain

$$
I^{3}=4 m_{f}\left[-\frac{1}{2} \ln \left(\frac{m_{f}}{\pi T}\right)-\frac{1}{2} \gamma_{E}+\mathcal{O}\left(\frac{m_{f}^{2}}{T^{2}}\right)\right] .
$$

In the above equation, $\gamma_{E}$ represents the Euler-Mascheroni constant. The temperatures achieved in the heavy ion collisions are far larger than the masses of the light flavors $(u$ and $d)$ i.e. $m_{f} \ll T$, so after dropping the term "O $\left(m_{f}^{2} / T^{2}\right)$ ", the eq. (A.96) becomes

$$
I^{3}=-2 m_{f}\left[\ln \left(\frac{m_{f}}{\pi T}\right)+\gamma_{E}\right] .
$$

Using the LLL approximation, we have done the $k_{\perp}$-integration in eq. (A.95) and the real part is obtained as

$$
\begin{aligned}
I^{\perp} & =\pi \int_{0}^{\left|q_{f} B\right|} d k_{\perp}^{2} \frac{1}{p_{\|}^{2}+m_{f}^{2}-k_{\perp}^{2}} \\
& =-\pi \ln \left(\frac{\left|q_{f} B\right|}{p_{\|}^{2}+m_{f}^{2}}\right) .
\end{aligned}
$$

After substituting the values of $I^{3}$ and $I^{\perp}$, the part containing the quark distribution function (A.95) becomes

$$
\Sigma_{n_{F}}\left(p_{\|}\right)=-\frac{2 g^{2} m_{f}}{3 \pi^{2}} \ln \left(\frac{\left|q_{f} B\right|}{p_{\|}^{2}+m_{f}^{2}}\right)\left[\ln \left(\frac{m_{f}}{\pi T}\right)+\gamma_{E}\right] .
$$

Now the medium part involving the gluon distribution function in eq. (A.93) is rewritten as

$$
\begin{aligned}
\Sigma_{n_{B}}\left(p_{\|}\right)= & -\frac{8 g^{2}}{3(2 \pi)^{3}} \int d^{2} k_{\perp} d k_{3} d k_{0}\left[J_{+}\left(\gamma^{0} k_{0}-\gamma^{3} k_{3}\right)-2 m_{f}\right] \\
& \times \frac{\delta\left(\left(p_{\|}-k_{\|}\right)^{2}-k_{\perp}^{2}\right) n_{B}\left(p_{0}-k_{0}\right)}{k_{0}^{2}-\omega_{k}^{2}} .
\end{aligned}
$$


To solve the above equation we first find the roots of the following equation,

$$
\left(p_{\|}-k_{\|}\right)^{2}-k_{\perp}^{2}=0
$$

then for small values of $k_{\perp}$ the above Dirac delta function is approximated as,

$$
\delta\left(\left(p_{\|}-k_{\|}\right)^{2}-k_{\perp}^{2}\right) \approx \frac{\left[\delta\left\{k_{0}-\left(p_{0}+p_{3}-k_{3}\right)\right\}+\delta\left\{k_{0}-\left(p_{0}-p_{3}+k_{3}\right)\right\}\right]}{2\left(p_{3}-k_{3}\right)} .
$$

Using the above Dirac delta function, after performing the $k_{0}, k_{\perp}$ and $k_{3}$ integrations, we get the following form of the self-energy (A.100),

$$
\begin{aligned}
\Sigma_{n_{B}}\left(p_{\|}\right)= & -\frac{2\left|q_{f} B\right| g^{2}}{3(2 \pi)^{2}} \int_{-\infty}^{+\infty} d k_{3} \frac{n_{B}\left(p_{3}-k_{3}\right)}{\left(p_{3}-k_{3}\right)}\left[\frac{J_{+} \gamma^{0} p_{0}-2 m_{f}}{\left(p_{0}+p_{3}-k_{3}\right)^{2}-\omega_{k}^{2}}\right. \\
& +\frac{J_{+} \gamma^{0}\left(p_{3}-k_{3}\right)}{\left(p_{0}+p_{3}-k_{3}\right)^{2}-\omega_{k}^{2}}-\frac{J_{+} \gamma^{3} k_{3}}{\left(p_{0}+p_{3}-k_{3}\right)^{2}-\omega_{k}^{2}}+\frac{J_{+} \gamma^{0} p_{0}-2 m_{f}}{\left(p_{0}-p_{3}+k_{3}\right)^{2}-\omega_{k}^{2}} \\
& \left.-\frac{J_{+} \gamma^{0}\left(p_{3}-k_{3}\right)}{\left(p_{0}-p_{3}+k_{3}\right)^{2}-\omega_{k}^{2}}-\frac{J_{+} \gamma^{3} k_{3}}{\left(p_{0}-p_{3}+k_{3}\right)^{2}-\omega_{k}^{2}}\right] \\
= & -\frac{2\left|q_{f} B\right| g^{2}}{3(2 \pi)^{2}} \sum_{i=1}^{6} L^{i}
\end{aligned}
$$

where $L^{i}$ 's are the imaginary quantities with the following values,

$$
\begin{aligned}
L^{1} & =\frac{i \pi\left(J_{+} \gamma^{0} p_{0}-2 m_{f}\right)}{2\left(p_{0}+p_{3}\right)}\left[\frac{\beta\left(a-p_{3}\right)-2}{2 \beta\left(p_{3}-a\right)^{2}}+\frac{n_{B}\left(a-p_{3}\right)}{a-p_{3}}\right], \\
L^{2} & =\frac{-i \pi J_{+} \gamma^{0}}{2\left(p_{0}+p_{3}\right)}\left[\frac{1}{\beta\left(p_{3}-a\right)}+n_{B}\left(a-p_{3}\right)\right], \\
L^{3} & =\frac{-i \pi J_{+} \gamma^{3}}{2\left(p_{0}+p_{3}\right)}\left[\frac{-2 a-\beta p_{3}\left(p_{3}-a\right)}{2 \beta\left(p_{3}-a\right)^{2}}+\frac{a n_{B}\left(a-p_{3}\right)}{a-p_{3}}\right], \\
L^{4} & =\frac{-i \pi\left(J_{+} \gamma^{0} p_{0}-2 m_{f}\right)}{2\left(p_{0}-p_{3}\right)}\left[\frac{\beta\left(b-p_{3}\right)-2}{2 \beta\left(p_{3}-b\right)^{2}}+\frac{n_{B}\left(b-p_{3}\right)}{b-p_{3}}\right], \\
L^{5} & =\frac{-i \pi J_{+} \gamma^{0}}{2\left(p_{0}-p_{3}\right)}\left[\frac{1}{\beta\left(p_{3}-b\right)}+n_{B}\left(b-p_{3}\right)\right], \\
L^{6} & =\frac{i \pi J_{+} \gamma^{3}}{2\left(p_{0}-p_{3}\right)}\left[\frac{-2 b-\beta p_{3}\left(p_{3}-b\right)}{2 \beta\left(p_{3}-b\right)^{2}}+\frac{b n_{B}\left(b-p_{3}\right)}{b-p_{3}}\right],
\end{aligned}
$$

where $a$ and $b$ are given by

$$
a=\frac{\left(p_{0}+p_{3}\right)^{2}-m_{f}^{2}}{2\left(p_{0}+p_{3}\right)}, b=\frac{\left(p_{0}-p_{3}\right)^{2}-m_{f}^{2}}{2\left(p_{3}-p_{0}\right)} .
$$


Thus the medium part involving gluon distribution function $\left(\Sigma_{n_{B}}\left(p_{\|}\right)\right)$is excluded from the real part of the quark self-energy.

In eq. (A.78), the medium part, containing the product of quark and gluon distribution functions,

$$
\begin{aligned}
\Sigma_{n^{2}}\left(p_{\|}\right)= & \frac{-8 g^{2} i}{3(2 \pi)^{2}} \int d^{2} k_{\perp} d^{2} k_{\|}\left[n_{F}\left(k_{0}\right) n_{B}\left(p_{0}-k_{0}\right)\right]\left[J_{+}\left(\gamma^{0} k_{0}-\gamma^{3} k_{3}\right)-2 m_{f}\right] \\
& \times\left[\delta\left(k_{\|}^{2}-m_{f}^{2}\right) \delta\left(\left(p_{\|}-k_{\|}\right)^{2}-k_{\perp}^{2}\right)\right]
\end{aligned}
$$

is imaginary, hence it is not included in the real part of the quark self-energy.

Now the total real part of the one-loop quark self-energy in a strong magnetic field is obtained by adding the vacuum (A.92) and the medium (A.99) parts,

$$
\begin{aligned}
\Sigma\left(p_{\|}\right)= & \frac{J_{+}\left(\gamma^{\|} \cdot p_{\|}\right) g^{2}}{12 \pi^{2}}\left[-1-\frac{\left|q_{f} B\right|}{m_{f}^{2}}\left\{\ln \left(\frac{\left|q_{f} B\right|}{m_{f}^{2}}\right)-1\right\}\right] \\
& +\frac{g^{2} m_{f}}{3 \pi^{2}}\left[1+\frac{J_{+}\left|q_{f} B\right|}{2 m_{f}^{2}}\left\{\ln \left(\frac{\left|q_{f} B\right|}{m_{f}^{2}}\right)-1\right\}\right] \\
& -\frac{2 g^{2} m_{f}}{3 \pi^{2}} \ln \left(\frac{\left|q_{f} B\right|}{p_{\|}^{2}+m_{f}^{2}}\right)\left[\ln \left(\frac{m_{f}}{\pi T}\right)+\gamma_{E}\right] .
\end{aligned}
$$

\section{B Free energy due to the quark contribution}

The free energy due to quark contribution is written as

$$
\mathcal{F}_{q}=-N_{c} N_{f} \int \frac{d^{2} p_{\perp}}{(2 \pi)^{2}} \int \frac{d^{2} p_{\|}}{(2 \pi)^{2}} \ln \left[\operatorname{det}\left(\gamma^{\|} \cdot p_{\|}-m_{f}-\Sigma\left(p_{\|}\right)\right)\right] .
$$

In the presence of a strong magnetic field in the $z$ direction, the momentum splits into the components transverse and longitudinal to the magnetic field, so the momentum integration also gets factorized into transverse and longitudinal parts and in SMF limit, the integrand becomes a function of the longitudinal momentum only. To solve eq. (B.106), 
we first write the quark self-energy (A.105) in a simplified version as

$$
\begin{aligned}
\Sigma\left(p_{\|}\right) & =J_{+}\left(\gamma^{\|} \cdot p_{\|}\right) \eta+J_{+} \Gamma+\xi+\Upsilon, \text { with } \\
\eta & =-\frac{g^{2}}{12 \pi^{2}}\left[1+\frac{\left|q_{f} B\right|}{m_{f}^{2}}\left\{\ln \left(\frac{\left|q_{f} B\right|}{m_{f}^{2}}\right)-1\right\}\right] \\
\Gamma & =\frac{g^{2}}{6 \pi^{2}} \frac{\left|q_{f} B\right|}{m_{f}}\left\{\ln \left(\frac{\left|q_{f} B\right|}{m_{f}^{2}}\right)-1\right\}, \\
\xi & =\frac{g^{2} m_{f}}{3 \pi^{2}} \\
\Upsilon & =-\frac{2 g^{2} m_{f}}{3 \pi^{2}} \ln \left(\frac{\left|q_{f} B\right|}{p_{\|}^{2}+m_{f}^{2}}\right)\left[\ln \left(\frac{m_{f}}{\pi T}\right)+\gamma_{E}\right] .
\end{aligned}
$$

The value of the determinant in eq. (B.106) is given by

$$
\begin{aligned}
\operatorname{det}\left[\gamma^{\|} \cdot p_{\|}-m_{f}-\Sigma\left(p_{\|}\right)\right]= & {\left[p_{\|}^{2}(1-2 \eta)^{2}-\left(m_{f}+2 \Gamma+\xi+\Upsilon\right)^{2}\right] } \\
& \times\left[p_{\|}^{2}-\left(m_{f}+\xi+\Upsilon\right)^{2}\right] .
\end{aligned}
$$

After substituting the determinant into eq. (B.106), we get

$$
\begin{aligned}
\mathcal{F}_{q}=-N_{c} N_{f} \int \frac{d^{2} p_{\perp}}{(2 \pi)^{2}} \int \frac{d^{2} p_{\|}}{(2 \pi)^{2}} \ln [ & \left\{p_{\|}^{2}(1-2 \eta)^{2}-\left(m_{f}+2 \Gamma+\xi+\Upsilon\right)^{2}\right\} \\
& \left.\times\left\{p_{\|}^{2}-\left(m_{f}+\xi+\Upsilon\right)^{2}\right\}\right] \\
\equiv-\frac{N_{c} N_{f}\left|q_{f} B\right|}{2 \pi}\left(I_{1}+I_{2}\right), &
\end{aligned}
$$

where the integral $I_{1}$ is written as

$$
I_{1}=\int \frac{d p_{0}}{2 \pi} \int \frac{d p_{3}}{2 \pi} \ln \left(p_{0}^{2}-p_{3}^{2}\right) .
$$

Using the imaginary-time formalism at finite temperature, the $I_{1}$ integral can be solved, where the continuous energy integrals are discretized into Matsubara frequency $\left(\omega_{n}\right)$ sums, i.e. $\int \frac{d p_{0}}{2 \pi} \rightarrow T \sum_{n}$. For quarks, $\omega_{n}=(2 n+1) \pi T$, where $n=0,1,2, \cdots$. After solving, we got the expression for $I_{1}$ as

$$
I_{1}=\frac{\pi T^{2}}{6}
$$


The $I_{2}$ integral is given by

$$
\begin{aligned}
& I_{2}=\int \frac{d^{2} p_{\|}}{2(2 \pi)^{2}} \ln \left[\left\{(1-2 \eta)^{2}-\frac{1}{p_{\|}^{2}}\left(m_{f}+2 \Gamma+\xi+\Upsilon\right)^{2}\right\}\left\{1-\frac{1}{p_{\|}^{2}}\left(m_{f}+\xi+\Upsilon\right)^{2}\right\}\right] \\
& =\int_{0}^{\left|q_{f} B\right|} \frac{d p_{\|}^{2}}{8 \pi} \ln \left[\left\{(1-2 \eta)^{2}-\frac{1}{p_{\|}^{2}}\left[m_{f}+2 \Gamma+\xi+\lambda \ln \left(\frac{\left|q_{f} B\right|}{p_{\|}^{2}+m_{f}^{2}}\right)\right]^{2}\right\}\right. \\
& \left.\times\left\{1-\frac{1}{p_{\|}^{2}}\left[m_{f}+\xi+\lambda \ln \left(\frac{\left|q_{f} B\right|}{p_{\|}^{2}+m_{f}^{2}}\right)\right]^{2}\right\}\right],
\end{aligned}
$$

where we have reexpressed the momentum-dependent term, $\Upsilon$ as

$$
\begin{aligned}
\Upsilon & =\lambda \ln \left(\frac{\left|q_{f} B\right|}{p_{\|}^{2}+m_{f}^{2}}\right), \text { with } \\
\lambda & =-\frac{2 g^{2} m_{f}}{3 \pi^{2}}\left[\ln \left(\frac{m_{f}}{\pi T}\right)+\gamma_{E}\right] .
\end{aligned}
$$

After plugging the integrals $I_{1}$ and $I_{2}$ in eq. (B.113), the free energy due to the quark contribution in a strong magnetic field is expressed as,

$$
\begin{aligned}
\mathcal{F}_{q}= & -\frac{N_{c} N_{f}\left|q_{f} B\right|}{4}\left[\frac{T^{2}}{3}+\int_{0}^{\left|q_{f} B\right|} \frac{d p_{\|}^{2}}{4 \pi^{2}} \ln \left[\left\{(1-2 \eta)^{2}\right.\right.\right. \\
& \left.-\frac{1}{p_{\|}^{2}}\left[m_{f}+2 \Gamma+\xi+\lambda \ln \left(\frac{\left|q_{f} B\right|}{p_{\|}^{2}+m_{f}^{2}}\right)\right]^{2}\right\} \\
& \left.\times\left\{1-\frac{1}{p_{\|}^{2}}\left[m_{f}+\xi+\lambda \ln \left(\frac{\left|q_{f} B\right|}{p_{\|}^{2}+m_{f}^{2}}\right)\right]^{2}\right\}\right] .
\end{aligned}
$$

\section{Integrals}

$$
\begin{aligned}
\int \frac{d^{3} \mathbf{p}}{(2 \pi)^{3}} \ln \left(\mathbf{p}^{2}+m_{q, D}^{2}\right) & =\mu^{2 \epsilon} \int \frac{d^{3-2 \epsilon} \mathbf{p}}{(2 \pi)^{3-2 \epsilon}} \ln \left(\mathbf{p}^{2}+m_{q, D}^{2}\right) \\
& =\frac{\mu^{2 \epsilon}}{2^{2-2 \epsilon} \pi^{\frac{3-2 \epsilon}{2}}} \frac{1}{\Gamma\left(\frac{3-2 \epsilon}{2}\right)} \int_{0}^{\infty} d \mathbf{p} \mathbf{p}^{2-2 \epsilon} \ln \left(\mathbf{p}^{2}+m_{q, D}^{2}\right) \\
& =-\frac{m_{q, D}^{3}}{3}\left(\frac{\mu}{2 m_{q, D}}\right)^{2 \epsilon}\left[\frac{1}{2 \pi}+\frac{4 \epsilon}{3}\right]
\end{aligned}
$$


where $\mu$ is an arbitrary mass parameter. Setting $\epsilon=0$, we get

$$
\begin{gathered}
\int \frac{d^{3} \mathbf{p}}{(2 \pi)^{3}} \ln \left(\mathbf{p}^{2}+m_{q, D}^{2}\right)=-\frac{m_{q, D}^{3}}{6 \pi} \\
\int \frac{d^{4} P}{(2 \pi)^{4}} \frac{\Pi_{\|}}{\mathbf{p}^{2}}=-\sum_{f} \int \frac{d^{3} \mathbf{p}}{(2 \pi)^{3}} \int \frac{d p_{0}}{2 \pi} \frac{g^{2}\left|q_{f} B\right| p_{3}^{2}}{8 \pi^{2} p_{\|}^{2} \mathbf{p}^{2}} \\
=-\frac{g^{2}}{8 \pi^{2}} \sum_{f}\left|q_{f} B\right| \int \frac{d^{3} \mathbf{p}}{(2 \pi)^{3}} T \sum_{n} \frac{1}{p_{0}^{2}-p_{3}^{2}} \frac{p_{3}^{2}}{\mathbf{p}^{2}}
\end{gathered}
$$

where the frequency sum is given by

$$
T \sum_{n} \frac{1}{p_{0}^{2}-p_{3}^{2}}=-\frac{1}{2 p_{3}}\left\{1+2 n_{B}\left(p_{3}\right)\right\} .
$$

Now eq. (C.122) becomes

$$
\int \frac{d^{4} P}{(2 \pi)^{4}} \frac{\Pi_{\|}}{\mathbf{p}^{2}}=\frac{g^{2}}{16 \pi^{2}} \sum_{f}\left|q_{f} B\right| \int \frac{d^{3} \mathbf{p}}{(2 \pi)^{3}} \frac{p_{3}}{\mathbf{p}^{2}}\left\{1+2 n_{B}\left(p_{3}\right)\right\} .
$$

For $p_{3}<T$, we solve the above integral and the simplified value is given by

$$
\int \frac{d^{4} P}{(2 \pi)^{4}} \frac{\Pi_{\|}}{\mathbf{p}^{2}}=\sum_{f} \frac{g^{2} T\left|q_{f} B\right| \Omega}{4(2 \pi)^{3}}
$$

where $\Omega$ is the ultraviolet cutoff parameter.

$$
\begin{aligned}
\int \frac{d^{4} P}{(2 \pi)^{4}} \frac{\Pi_{\|}^{2}}{2 \mathbf{p}^{4}} & =\frac{g^{4}}{128 \pi^{4}} \sum_{f}\left|q_{f} B\right|^{2} \int \frac{d^{3} \mathbf{p}}{(2 \pi)^{3}} \int \frac{d p_{0}}{2 \pi} \frac{p_{3}^{4}}{\mathbf{p}^{4} p_{\|}^{4}} \\
& =\frac{g^{4}}{128 \pi^{4}} \sum_{f}\left|q_{f} B\right|^{2} \int \frac{d^{3} \mathbf{p}}{(2 \pi)^{3}} \frac{p_{3}^{4}}{\mathbf{p}^{4}} T \sum_{n} \frac{1}{\left(p_{0}^{2}-p_{3}^{2}\right)^{2}} .
\end{aligned}
$$

The frequency sum in the above equation is performed as follows.

$$
\begin{aligned}
T \sum_{n} \frac{1}{\left(p_{0}^{2}-p_{3}^{2}\right)^{2}} & =T \sum_{n} \frac{1}{2 p_{3}} \frac{\partial}{\partial p_{3}}\left(\frac{1}{p_{0}^{2}-p_{3}^{2}}\right) \\
& =\frac{1}{4 p_{3}^{3}}\left\{1+2 n_{B}\left(p_{3}\right)\right\}+\frac{1}{2 T p_{3}^{2}}\left\{1+n_{B}\left(p_{3}\right)\right\} n_{B}\left(p_{3}\right) .
\end{aligned}
$$

Substituting the frequency sum in eq. (C.126), we obtain the terms involving infrared divergence. So, in solving the integration, we use cutoff regularization method to discard the divergence and thus, eq. (C.126) is simplified into

$$
\int \frac{d^{4} P}{(2 \pi)^{4}} \frac{\Pi_{\|}^{2}}{2 \mathbf{p}^{4}}=\sum_{f} \frac{g^{4} T\left|q_{f} B\right|^{2}}{128 \pi^{2}(2 \pi)^{3} \zeta}
$$

where $\zeta$ represents the infrared cutoff parameter. 


\section{References}

[1] K. Tuchin, Phys. Rev. C 83, 017901 (2011).

[2] K. Fukushima and J. M. Pawlowski, Phys. Rev. D 86, 076013 (2012).

[3] G. S. Bali, F. Bruckmann, G. Endrődi, S. D. Katz and A. Schäfer, JHEP 1408, 177 (2014).

[4] G. Endrődi, JHEP 1507, 173 (2015).

[5] J. O. Andersen, W. R. Naylor and A. Tranberg, Rev. Mod. Phys. 88, 025001 (2016).

[6] S. Rath and B. K. Patra, JHEP 1712, 098 (2017).

[7] K. Fukushima, D. E. Kharzeev and H. J. Warringa, Phys. Rev. D 78, 074033 (2008).

[8] D. E. Kharzeev, L. D. McLerran and H. J. Warringa, Nucl. Phys. A 803, 227 (2008).

[9] H. van Hees, C. Gale and R. Rapp, Phys. Rev. C 84, 054906 (2011).

[10] C. Shen, U. W. Heinz, J.-F. Paquet and C. Gale, Phys. Rev. C 89, 044910 (2014).

[11] K. Tuchin, Phys. Rev. C 88, 024910 (2013).

[12] K. A. Mamo, JHEP 1308, 083 (2013).

[13] R. K. Mohapatra, P. S. Saumia and A. M. Srivastava, Mod. Phys. Lett. A 26, 2477 (2011).

[14] V. Braguta, M. N. Chernodub, V. A. Goy, K. Landsteiner, A. V. Molochkov and M. I. Polikarpov, Phys. Rev. D 89, 074510 (2014).

[15] M. N. Chernodub, A. Cortijo, A. G. Grushin, K. Landsteiner and M. A. H. Vozmediano, Phys. Rev. B 89, 081407 (R) (2014).

[16] D. E. Kharzeev and Dam T. Son, Phys. Rev. Lett. 106, 062301 (2011).

[17] N. Mueller and J. M. Pawlowski, Phys. Rev. D 91, 116010 (2015).

[18] V. P. Gusynin, V. A. Miransky and I. A. Shovkovy, Phys. Rev. Lett. 73, 3499 (1994).

[19] A. Haber, F. Preis, and A. Schmitt, Phys. Rev. D 90, 125036 (2014).

[20] M. Strickland, V. Dexheimer and D. P. Menezes, Phys. Rev. D 86, 125032 (2012). 
[21] G. Basar, D. Kharzeev, D. Kharzeev and V. Skokov, Phys. Rev. Lett. 109, 202303 (2012).

[22] A. Ayala, J. D. Castano-Yepes, C. A. Dominguez and L. A. Hernandez, EPJ Web Conf. 141, 02007 (2017).

[23] J. C. D’Olivo, J. F. Nieves and S. Sahu, Phys. Rev. D 67, 025018 (2003).

[24] A. K. Ganguly, S. Konar and P. B. Pal, Phys. Rev. D 60, 105014 (1999).

[25] K. Tuchin, Phys. Rev. C 87, 024912 (2013).

[26] D. N. Kabat, K.-M. Lee and E. J. Weinberg, Phys. Rev. D 66, 014004 (2002).

[27] O. Bergman, G. Lifschytz and M. Lippert, JHEP 05, 007 (2008).

[28] K. Sato and T. Tatsumi, Prog. Theor. Phys. Suppl. 174, 177 (2008).

[29] T. Steinert and W. Cassing, Phys. Rev. C 89, 035203 (2014).

[30] A. N. Tawfik and N. Magdy, Phys. Rev. C 90, 015204 (2014).

[31] K. Kamikado, T. Kanazawa, JHEP 01, 129 (2015).

[32] U. Gürsoy, D. Kharzeev and K. Rajagopal, Phys. Rev. C 89, 054905 (2014).

[33] V. Roy and S. Pu, Phys. Rev. C 92, 064902 (2015).

[34] Y. Hirono, T. Hirano and D. E. Kharzeev, arXiv:1412.0311 [hep-ph].

[35] Long-Gang Pang, Gergely Endrődi and Hannah Petersen, Phys. Rev. C 93, 044919 (2016).

[36] V. Roy, S. Pu, L. Rezzolla and D. H. Rischke, Phys. Rev. C 96, 054909 (2017).

[37] S. Pu, V. Roy, L. Rezzolla and D. H. Rischke, Phys. Rev. D 93, 074022 (2016).

[38] J. Schwinger, Phys. Rev. 82, 664 (1951).

[39] Wu-yang Tsai, Phys. Rev. D 10, 2699 (1974).

[40] E. J. Ferrer, V. de la Incera and X. J. Wen, Phys. Rev. D 91, 054006 (2015).

[41] M. A. Andreichikov, V. D. Orlovsky and Yu. A. Simonov, Phys. Rev. Lett. 110, 162002 (2013).

[42] V. P. Gusynin and A. V. Smilga, Phys. Lett. B 450, 267 (1999). 
[43] E. Braaten and R. D. Pisarski, Phys. Rev. Lett. 64, 1338 (1990).

[44] E. Braaten and R. D. Pisarski, Phys. Rev. D 42, 2156 (1990).

[45] R. Kobes, G. Kunstatter and K. Mak, Phys. Rev. D 45, 4632 (1992).

[46] K. Hattori and D. Satow, Phys. Rev. D 97, 014023 (2018).

[47] K. Hattori and K. Itakura, Annals Phys. 330, 23 (2013).

[48] A. Ayala, C. A. Dominguez, S. Hernandez-Ortiz, L. A. Hernandez, M. Loewe, D. M. Paret and R. Zamora, arXiv:1805.07344 [hep-ph].

[49] A. Ayala, C. A. Dominguez, S. Hernandez-Ortiz, L. A. Hernandez, M. Loewe, D. M. Paret and R. Zamora, Phys. Rev. D 98, 031501 (2018).

[50] K. Fukushima, K. Hattori, H-U. Yee and Y. Yin, Phys. Rev. D 93, 074028 (2016).

[51] M. Hasan, B. Chatterjee and B. K. Patra, Eur. Phys. J. C 77, 767 (2017).

[52] B. Singh, L. Thakur and H. Mishra, Phys. Rev. D 97, 096011 (2018).

[53] J. O. Andersen, E. Braaten, E. Petitgirard and M. Strickland, Phys. Rev. D 66, 085016 (2002).

[54] G. S. Bali, F. Bruckmann, G. Endrődi, and A. Schäfer, Phys. Rev. Lett. 112, 042301 (2014).

[55] C. Bonati, M. D’Elia, M. Mariti, F. Negro and F. Sanfilippo, Phys. Rev. D 89, 054506 (2014).

[56] L. Levkova and C. DeTar, Phys. Rev. Lett. 112, 012002 (2014).

[57] G. Endrődi, JHEP 04, 023 (2013).

[58] B. -J. Schaefer, M. Wagner, J. Wambach, PoS CPOD2009, 017 (2009).

[59] V. Koch, J. Phys. G: Nucl. Part. Phys. 35, 104030 (2008).

[60] Min He, Yu Jiang, Wei-Min Sun and Hong-Shi Zong, Phys. Rev. D 77, 076008 (2008).

[61] Lei Chang, Yu-xin Liu, Craig D. Roberts, Yuan-mei Shi, Wei-min Sun and Hong-shi Zong, Phys. Rev. C 79, 035209 (2009).

[62] K. Tuchin, Adv. High Energy Phys. 2013, 490495 (2013).

[63] S. Rath and B. K. Patra, arXiv:1901.03855 [hep-ph]. 
[64] W. Israel and J. M. Stewart, Annals Phys. 118, 341 (1979).

[65] A. Muronga, Phys. Rev. Lett. 88, 062302 (2002).

[66] R. Baier, P. Romatschke, and U. A. Wiedemann, Phys. Rev. C 73, 064903 (2006).

[67] P. Kovtun, D. Son and A. Starinets, Phys. Rev. Lett. 94, 111601 (2005).

[68] P. Arnold, G. D. Moore and L. G. Yaffe, JHEP 11, 001 (2000).

[69] A. Kisiel, W. Broniowski, M. Chojnacki and W. Florkowski, Phys. Rev. C 79, 014902 (2009).

[70] L. Dolan and R. Jackiw, Phys. Rev. D 9, 3320 (1974). 